\title{
Rapid Oscillations in Cataclysmic Variables
}

\author{
Brian Warner \\ Department of Astronomy, University of Cape Town, Rondebosch, 7700 South Africa \\ warner@physci.uct.ac.za
}

Received __; accepted _ 


\begin{abstract}
I give an overview of the rich phenomenology of dwarf nova oscillations (DNOs) and Quasi-periodic Oscillations (QPOs) observed in cataclysmic variable stars $(\mathrm{CVs})$. The favoured interpretation of these rapid brightness modulations $(3->1000 \mathrm{~s}$ time scales $)$ is that they are magnetic in nature - magnetically channelled accretion from the inner accretion disc for DNOs and possible magnetically excited travelling waves in the disc for QPOs. There is increasing evidence for the magnetic aspects, which extend to lower fields the well known properties of strong field (polars) and intermediate strength field (intermediate polars) CVs - The result is that almost all CVs show the presence of magnetic fields on their white dwarf primaries - though for many the intrinsic field may be locally enhanced by the accretion process itself.

There are many parallel behaviours with the QPOs seen in X-Ray binaries, with high and low frequency X-Ray QPOs resembling respectively the DNOs and QPOs in CVs.
\end{abstract}

Subject headings: cataclysmic variables, stars: binaries: close

\title{
1. INTRODUCTION
}

The discovery by Merle Walker (1956) on 9 July 1954 of a 71 s modulation in the light curve of DQ Her, the remnant of nova Herculis 1934, gave notice that cataclysmic variables $(\mathrm{CVs})$ not only are binaries of short orbital period, they also are capable of producing periodic phenomena on what, in 1954, was of unprecedentedly short time scales. The rapidity and high stability of the modulation in DQ Her is now attributed to rotation of the white dwarf primary, and was the forerunner of the class of CVs known as intermediate 
polars (IPs), in which the magnetic field of the primary channels gas from the inner edge of an accretion disc onto spots or arcs on the primary, and of which about 32 are now known (Kuulkers et al. 2003). In the case of DQ Her the optical oscillations have been shown to be due to anisotropic radiation from the primary (located probably at two accretion hotspots, so the rotation period is actually $142 \mathrm{~s}$ ), sweeping across the accretion disc, deduced from observed phase changes in eclipse of the continuum modulation and from the wavelength-dependence of pulsation phase in the emission lines (Warner et al. 1972; Patterson, Robinson \& Nather 1978; Chanan, Nelson \& Margon 1978; Martell et al. 1995; Silber et al. 1996).

In the early searches for further examples of the DQ Her phenomenon (see Warner (1988) for a history of the application of high speed photometry to CVs) only one more nova, V533 Her, was added (Patterson 1979). However, the same time scale phenomenon, albeit of much lower stability and usually of very low amplitude, was found (Warner \& Robinson 1972) to be commonly present in dwarf novae during outburst and also in nova-like variables, i.e., in CVs of high rates of mass transfer $(\dot{M})$. These modulations, with periods initially observed in the range $8-40 \mathrm{~s}$, became known as Dwarf Nova Oscillations (DNOs). In the optical they are usually of such low amplitude that they appear only in Fourier transforms, but occasionally they reach a few percent amplitude and can be seen directly in the light curve (Fig. 1). In almost all cases they are highly sinusoidal in pulse profile. There is no apparent correlation between amplitude of DNOs and orbital inclination.

The study of such rapid modulations in CV light curves used to necessitate the use of relatively inefficient photomultipliers, but the advent of high quantum efficiency CCDs, and the ability to window and bin their pixels, has made possible studies to much fainter levels, and in particular in crowded fields. An example of such a design, and its applications, is 
given in O’Donoghue (1995).

Longer time scale modulations, of large amplitude but much lower coherence, were identified in CV light curves by Patterson, Robinson \& Nather (1977) and are commonly called Quasi-Periodic Oscillations (QPOs). As will be discussed below, these may have more than one time scale and physical origin.

Recently, a new type of DNO has been recognised (Warner, Woudt \& Pretorius 2003; hereafter WWP), similar to the DNOs but having periods typically a factor of about four greater. These can co-exist with the DNOs and QPOs, and may at times be mistaken for ordinary DNOs.

I will examine the general properties of each of these kinds of short time scale modulations. A general review of the literature on oscillations in CVs up to 1995 can be found in Warner (1995a). I omit from discussion here the rapid oscillations that are observed at $\sim 1 \mathrm{~Hz}$ in the accretion columns of strongly magnetic CVs (polars) - e.g., Larsson (1992).

\section{DNOs}

From the time of their discovery it was recognised that, unlike DQ Her, which has $Q=\left|\frac{d P}{d t}\right|^{-1} \sim 10^{12}$, DNOs are low $Q$ modulations. On time scales of hours their periods can change by many seconds, but at other times are stable to milliseconds for many hours, giving $10^{3}<Q<10^{7}$. Two time scales of variation are known. The first, on an outburst time scale, is a period-luminosity relationship, connecting DNO period to optical brightness, but not in a single-valued manner, thus producing "banana loops" in a diagram of $P_{D N O}$ versus $m_{v}$ (Patterson 1981). Although it was early hypothesised that $P_{D N O}$ would be found to be simply related to accretion luminosity (Bailey 1980), and that this would produce 
such loops because of the observed time delay between bolometric and optical luminosities (Hassall et al. 1983; Warner 1995b), it was long before this could be directly demonstrated as a monotonic relationship between $P_{D N O}$ and EUV luminosity (which is a good monitor of bolometric accretion luminosity: Mauche 1996). It was even longer before simultaneous observations of DNOs in the optical and EUV showed identical, in phase, modulations (Mauche \& Robinson 2001).

Although covering a smaller range of luminosities, the period-luminosity relationship has been demonstrated for nova-likes (Warner, O’Donoghue \& Allen 1985). It would be of interest to study any DNOs in nova-likes that go into states of low $\dot{M}$ (i.e., the VY Scl stars).

The second time scale of variation is very short: even when the DNOs are at their most coherent, changes are seen as sudden small jumps in period (typically $\sim 0.03$ s, i.e. $\sim$ $0.1 \%$ ) that interrupt times of comparative stability lasting for thousands of seconds (Fig. 2). There is evidence that DNOs are most stable at maximum of outburst but that their stability (i.e., coherence length) progressively decreases late in outburst (e.g. Hildebrand, Spillar \& Stiening 1981). The rarity with which any optical DNOs are seen at maximum light in VW Hyi, and their low amplitude $(\sim 1 \mathrm{mmag})$ when observed (Woudt \& Warner 2002a: hereafter WW2a), could indicate that it is high stability that is rare, and that the usually employed Fourier transform techniques are inadequate for detecting drifting signals. Similarly, van der Woerd et al. (1987) were successful in detecting DNOs on only two occasions during extensive soft X-Ray observations of 7 outbursts of VW Hyi, where the modulation is large $(\sim 15 \%)$ when present.

The night-to-night change from easy detectability to complete absence of DNOs in nova-likes (e.g. UX UMa, where DNOs are found in only about half of the optical and HST observations: Nather \& Robinson (1974); Knigge et al. (1998)) also indicate cessation 
rather than reduced coherence. The changes of amplitude of DNOs over a few hours often include disappearance and reappearance.

In addition to the abrupt changes in period, which are not accompanied by any noticeable change in system luminosity, there are short-lived phase excursions that appear to have no effect on the underlying trend in period. When best defined, the DNOs show the presence of an underlying clock with abrupt changes of period, on which the phase noise seems never to cause loss of knowledge of phase (Warner \& Brickhill 1978; Jones \& Watson 1992). However, the increasing frequency of the phase and period changes produces reduced coherence in late phases of outbursts. These short time scale variations are demonstrated in Fig. 2.

When sufficient DNO coherence occurs in eclipsing dwarf novae or nova-likes, phase and amplitude variations can be measured during the eclipses. These show that, as in DQ Her, DNOs are caused by "beams" of radiation carried round by rotation of the primary, (Nather \& Robinson 1974; Patterson 1981; Petterson 1980). Confirmation comes from Keck time-resolved spectroscopy of V2051 Oph, which has a pulse phase in its emission lines that is wavelength dependent similar to that of DQ Her (Steeghs et al. 2001).

An inventory of DNO observations ${ }^{1}$ is given in Table 1 . There are about 50 CVs in which standard DNOs have been detected, plus a few in which the nature of the DNOs

\footnotetext{
${ }^{1}$ In many cases there has been only one observation of a DNO in a given star, but its persistence for an hour or more is sufficient evidence for its existence. The full range of period is in most cases not yet determined, and therefore the order of stars in Table 1 (where in the first part they are listed by increasing minimum observed period) is to some extent arbitrary. In a few cases of very low amplitude, short duration of appearance, or other reason, I have omitted published claims of DNO detections.
} 
is not yet determined. It is notable that none of the classic IPs appears in this list (an instructive exception is GK Per, see Section 4) - despite much high speed photometry none of these has been reported to have modulations at periods shorter than their primary spin-related periods.

\section{LONGER PERIOD DNOs (lpDNOs)}

It has recently been realised, from observing the literature as well as the sky, that there is another class of DNOs, showing coherence levels similar to DNOs but not following the same period-luminosity relationship - in fact, their periods may be independent of $\dot{M}$ (WWP). We have called these 'longer period DNOs' (lpDNOs); they have periods typically about four times greater than those of DNOs and can coexist with the latter. An example of this is shown in Fig. 3. They are mentioned, but without explanation, in a number of early papers on DNOs - specific examples are in VW Hyi, where a modulation with period averaging $\sim 88 \mathrm{~s}$ (in addition to $\sim 28-36 \mathrm{~s}$ DNOs) was seen on seven nights of an outburst as the system decreased in brightness from magnitude 10.6 to 13.7 (Haefner, Schoembs \& Vogt 1979; examples of similar, but rare, periodicities in VW Hyi are given by WWP); SS Cyg, where $\sim 33$ s modulation was present in addition to $10 \mathrm{~s}$ DNOs (Robinson \& Nather 1979; Patterson 1981); HT Cas and AH Her, where 100 s modulations were seen (the former even in quiescence) but where normal DNOs have much shorter periods (Patterson 1981).

lpDNOs have now been observed in about $17 \mathrm{CVs}$ (Table 1); these include some reclassifications, and there may be other cases where DNOs have mistaken identity. No studies of phase shifts during eclipse have yet been made, but the existence of double lpDNOs (Section 4.1) indicates that a rotating beam model is applicable and therefore phase shifts should occur. 
As with DNOs, the lpDNOs are not always present; on average they have slightly larger amplitudes than DNOs and are more often seen directly in the light curve - see Fig. 4. For this reason some previously observed lpDNOs were classified as QPOs.

Comparison of the amplitude and phase changes of DNOs and lpDNOs when both are simultaneously present shows that they generally behave independently (WWP).

Table 1 includes four AM CVn stars - helium-transferring CVs with ultra-short orbital periods. The detection of the various types of rapid modulation in AM CVn stars is made difficult by the presence of strong harmonics to the main orbital/superhump brightness variations. (These harmonics are not independent oscillations in the system - they appear in Fourier transforms merely from the highly nonsinusoidal shapes of the principal modulations.)

Nevertheless, V803 Cen has long been known to possess a modulation at $\sim 178 \mathrm{~s}$ (O’Donoghue, Menzies \& Hill 1987) of unknown origin. It is seen when V803 Cen has a

high $\dot{M}$, but not during low states, its period appears independent of luminosity, and there are phase variations on time scales of hours, similar to those of DNOs (WWP). These are the characteristics of lpDNOs, rather than, e.g. possible pulsations of the primary. No ordinary DNOs have yet been detected in V803 Cen, but its compatriot CR Boo has shown the full set of DNO, lpDNO and QPO oscillations (WWP) and the type star AM CVn has shown both ordinary DNOs and two periodicities in the QPO range.

\section{QUASI-PERIODIC OSCILLATIONS}

Quasi-periodic modulations have orders of magnitude less coherence than DNOs. Because of this they are difficult to recognise in Fourier transforms - their power is spread over a wide band of frequency. They typically have $Q \sim 5-20$. An example of an unusually 
coherent train of 11 cycles of a QPO is shown in Fig. 5; a Fourier transform of this light curve shows that $19.4 \mathrm{~s}$ DNOs with a mean amplitude of 3.8 mmag were also present.

In principle there may be more than one kind of variation, e.g. almost constant period but with frequent large phase changes, with perhaps a clear growth and decay in amplitude between the phase jumps, or constantly varying period and amplitude over a limited range. There has as yet been no study or classification of such modulations; their analysis is hampered by the general stochastic flickering, arising from accretion processes, that is usually present in CVs (though here again some reclassification may be necessary - some of the flickering and flaring commented on in the CV literature has a QPO look about it). More refined definitions and analyses will probably reveal that QPOs are more common than hitherto realised - at present we accept only the QPOs in a light curve that are obvious to the eye.

A glance down the column listing QPO periods in Table 1 will indicate the evident existence of two time scales of QPO. In a few cases (e.g. WX Hyi, VZ Pyx, OY Car, perhaps V2051 Oph) two quite different periods of QPOs have been observed. For those systems where DNOs and/or lpDNOs are seen there are often QPOs at $\sim 15$ times the DNO period or, equivalently, $\sim 4$ times the lpDNO period. Apart from these there is a large number of observations of quasi-periodic modulations with periods in the range 1000 - $3000 \mathrm{~s}$ (note that GK Per is an exception here - its $5000 \mathrm{~s}$ QPO is $\sim 15$ times its optical DNO period). I have to distinguish between these two kinds of QPO here, but am reluctant to introduce yet more nomenclature, and so will simply discuss separately those that are related to $\mathrm{DNO} / \mathrm{lpDNO}$ periods and those that are not. 


\subsection{DNO-related QPOs}

A valuable clue to the nature of these QPOs is given by the existence of "double DNOs". Pairs of DNOs with small separations in period were found in V3885 Sgr (29.08 and 30.15 s: Hesser, Lasker \& Osmer 1974), V2051 Oph (29.77 and 28.06 s: Steeghs et al. 2001), OY Car (17.94 and 18.16 s: Marsh \& Horne 1998), V436 Cen (19.45 and 20.20 s: WWP), EC2117-54 (many examples, typically 22.10 and 23.27 s: WWP), CN Ori (11.23 and 12.10 s: WWP) and VW Hyi (see below: WW2a) - and there is the case of WZ Sge, which shows stable modulations at 27.87 and/or 28.95 s even in quiescence (Table 1), which I will discuss in Section 9.

In many of these cases the beat period between the double DNOs corresponds to the period of a QPO present at the same time - including WZ Sge (Warner \& Woudt 2002b: hereafter WW2b). In Fig. 6 the mean pulse profiles of two DNO components and the accompanying QPO are illustrated. An example of a double lpDNO, split at the QPO frequency, has been found in VW Hyi (WWP).

Another clear example of an interrelationship between DNOs and QPOs is given by the light curve of VW Hyi for 5 Feb 2000 (Fig. 7), obtained at the end of outburst when large amplitude DNOs and QPOs typically occur in this star. The fall in brightness by a factor of about two during the observation caused steady increases in period for both the DNOs and the QPOs - the first time such an evolution of QPO period had been seen. The ratio $P_{Q P O} / P_{D N O}$ remained at about 15 during this evolution (Fig. 8). This is generalised in Section 10. 


\subsection{Other QPOs}

Even among the remaining QPOs there may be two or more distinct types. Many of the CVs listed in Table 1 with periods $\sim 1000 \mathrm{~s}$ are suspected by Patterson et al. (2002b) to be generated by IP structures - as in the case of GK Per where the underlying $351 \mathrm{~s}$ rotation of the primary is seen as $\sim 380 \mathrm{~s}$ modulation caused by reprocessing of the rotating beam off a varying period QPO source. The periods are characteristic of the "canonical" IPs, which have rotation periods $\sim 15$ min and binary periods $\sim 4 \mathrm{~h}$. Included among these systems are LS Peg and V795 Her, both of which show polarization modulated at the optical periods (Rodriquez-Gil et al. 2001, 2002), but which have not yet been proved to have the high stability required for definite IP status.

For these systems, therefore, the evidence is accumulating that their QPOs are another manifestation of magnetic primaries - and are occurring in IPs for which large amplitude coherent optical and X-Ray modulations are in some way suppressed - perhaps by high $\dot{M}$. These will therefore probably add directly to the tally of IPs.

But, anticipating the model developed in Section 6, a CV cannot have DNOs (and/or lpDNOs) and be an IP - the magnetic field is either strong enough to anchor the exterior of the white dwarf to the interior, or it is not - and this exclusivity is indeed demonstrated by the absence of any observed DNOs in known IPs. So we are left with at least a few CVs for which the QPOs cannot have a direct connection with rotation of the primary. In Table 1 these are the systems WX Hyi, VZ Pyx, OY Car and V2051 Oph, already mentioned above, and there may be others in which only QPOs have so far been detected. The time scales of some of these modulations are comparable with the rotation periods at the outer edges of a high $\dot{M}$ accretion discs (which are $\sim 0.20 P_{\text {orb }}$ - equation 8.6 of Warner 1995a), but no definite models accompany this speculation.

Another possible source of brightness variation is modulation of $\dot{M}$ from the secondary, 
caused by non-radial oscillations of the secondary in a manner similar to that of the $\sim 5$ min oscillations of the Sun. Small amplitude variations in radius of the secondary would be amplified in $\dot{M}$ by the great sensitivity of Roche lobe overflow.

\section{X-RAY OBSERVATIONS}

CVs, even nominally 'non magnetic' ones, are usually detectable in X-Rays: the low $\dot{M}$ systems have hard X-Ray emission and the high $\dot{M}$, optically thick, systems have soft X-Rays. A transition from hard to soft and back is seen during a dwarf nova outburst.

Table 2 lists X-Ray observations of DNOs and QPOs in CVs - it includes only the lower $Q$ modulations, i.e., it omits IPs. The best-studied systems are SS Cyg, U Gem and VW Hyi, which are close enough to the Sun for soft X-Rays to be received almost unabsorbed by interstellar gas and are thus very strong sources during outbursts. There appears to be a mixture of DNOs, lpDNOs and QPOs, but VW Hyi and U Gem in particular have full sets (note that in the case of VW Hyi the DNOs were observed at maximum but the QPOs were at the very end of outburst, so the ratio $P_{Q P O} / P_{D N O}$ is not close to the value 15 seen in the optical). Where there is an overlap, it is evident that individual stars show the same modulation time scales in optical and X-Ray.

The phase behaviour of DNOs is the same in X-Rays as in the optical. The most detailed study is that of Jones \& Watson (1992); one of their results is shown in Fig. 9, which should be compared with Fig. 2. The amplitude of modulation in this case averaged about $35 \%$ but at times reached nearly 100\%. Such large amplitudes are commonly seen in soft X- Ray DNOs.

Table 2 shows that ordinary DNOs seen during dwarf nova outbursts occur in soft X-Rays but not hard X-Rays. This is most notable in the comprehensive study by Wheatley, 
Mauche \& Mattei (2003: hereafter WMM) of an outburst in SS Cyg. The few observed hard X-Ray DNOs have all occurred at quiescence. WMM conclude that the source of the DNOs must lie in the optically thick boundary layer at the surface of the primary. Although 'residual' hard X-Rays are observed during outburst, these are not modulated as DNOs but this is in accordance with the interpretation of these X-Rays as being coronal in origin $(\mathrm{WMM})$.

On the other hand, modulations at QPO periods are seen in hard X-Rays, most strongly in VW Hyi just as the hard X-Rays are turning on at the end of an outburst (Wheatley et al. 1996) and in SS Cyg during the turn-off phase of hard X-Rays after the start of outburst and again when they turn on at the end of outburst (WMM). These phases correspond to the transitions between optically thick and optically thin discs, which themselves are governed by $\dot{M}$ in the inner disc. The QPOs themselves are not accompanied by any hardness variations, thus eliminating temperature variations or photoelectric absorption variations as the source of the oscillations - but it does allow quasi-periodic occultation by a source that is completely opaque at X-ray energies (WMM).

\section{STATISTICAL VERSUS PHYSICAL MODELS}

A number of statistical analyses of DNO light curves have been undertaken. Early models included a damped harmonic oscillator excited by white noise (Robinson \& Nather 1979; Hildebrand, Spillar \& Stiening 1981), and a sinusoidal oscillator exercising a random walk in phase (Horne \& Gomer 1980; Cordova et al. 1980, 1984). One problem with such approaches is that although they may deliver quantitative values of statistical parameters these do not necessarily lead to any physical insight. A worse problem arises when it is seen that, for the best quality data, such analyses are inappropriate - e.g., Jones \& Watson (1992) show that the random walk model does not represent the behaviour of the soft 
X-Ray DNOs of SS Cyg. But that should be no surprise given the systematic behaviour seen in the phase diagrams (Figs. 2 and 9).

A more profitable approach, therefore, is to start with the observed properties and try to envisage a model that can reproduce them. In presenting what I think is a viable model I am influenced by the following facts:

- At least $10 \%$ of single white dwarfs have magnetic fields $>2 \times 10^{6} \mathrm{G}$, and the limited studies with sensitivities down to $\sim 3 \times 10^{4} \mathrm{G}$ suggest that the total may be significantly higher (Liebert, Bergeron \& Holberg 2003). At least 25\% of white dwarfs in CVs have detectable or directly inferable fields (i.e. they are polars or IPs), despite the fact that the current lower limit for detection in these systems is $\sim 7 \times 10^{6}$ G (Wickramasinghe \& Ferrario 2000). But here there may be a selection effect operating: most strongly magnetic CVs have been found from X-Ray surveys, which probe to greater distances than extant optical surveys (though the Sloan Digital Sky Survey may eventually remove this bias). On the other hand, at least some of the high $\dot{M}$ IPs avoided detection until recently (e.g. the SW Sex systems LS Peg and V795 Her). It has been suggested that in the common envelope phase magnetic fields are generated and amplified within the differentially rotating envelope (Regos \& Tout 1995), so it would not be surprising to find that CV primaries are systematically more magnetic than isolated white dwarfs.

- Given this we may suspect that, not only are there many primaries in CVs with fields $<7 \times 10^{6} \mathrm{G}$, they may even constitute a dominant fraction of the apparently "non magnetic" majority. The DNO model that I favour is based on this expected extension of the distribution of magnetic fields of primaries to values lower than those in IPs. 


\section{A MAGNETIC MODEL FOR DNOs and lpDNOs}

The observed period-luminosity relationship for DNOs prompted Paczynski (1978) to suggest that magnetically channelled accretion was responsible, but onto an equatorial belt that slips on the surface of the primary, rather than onto the body of the primary itself. This was developed by Warner (1995b) and more recently in WW2b. Direct evidence for long-lived, rapidly rotating equatorial belts on the primaries of dwarf novae after outburst has come from HST observations (Sion et al. 1996; Gänsicke \& Beuermann 1996; Cheng et al. 1997; Szkody et al. 1998; Sion \& Urban 2002), where spectra are found to be composite, comprising a white dwarf in relatively slow rotation plus a hot belt rotating at a rate comparable to the Keplerian velocity at the surface of the primary.

I will describe the model briefly, and show how it relates to the principal observed properties of DNOs, lpDNOs and QPOs.

An accretion torque applied to the surface of a star can only be communicated to the bulk of the star if the viscosity of the interior of the star is large enough. Degenerate material is notoriously slippery, so a white dwarf behaves like a solid body only if it is permeated by a strong enough magnetic field (Durisen 1973). Strong enough' turns out to

be $B>1 \times 10^{5} \mathrm{G}$ (Katz 1975, WW2b). For accretion to be magnetically controlled near the surface of the primary $B$ must also be strong enough - depending on $\dot{M}$ and the geometry of the field. For $\dot{M} \sim 5 \times 10^{17} \mathrm{~g} \mathrm{~s}^{-1}$, probably the maximum reached in dwarf novae in outburst, the requirement (for a multipole field) is $B>2 \times 10^{4} \mathrm{G}$ (equation 16 of WW2b).

There is therefore a window of opportunity - even high $\dot{M}$ accretion onto low field primaries can be magnetically controlled by whatever field the freely slipping equatorial belt has. The field within the belt itself will be enhanced during accretion, by differential shearing of the field lines, which aids magnetic channelling; this may also explain why dwarf novae in quiescence in general do not show DNOs. CVs with very low intrinsic fields should 
never show DNOs: several dwarf novae and nova-likes have been extensively observed with high time resolution without any sign of DNOs (Patterson (1981) and Warner (1995a) list examples) - these constitute the genuine non-magnetic CVs.

The model is therefore similar to that for an IP, but is of low $Q$ : the mass of the belt accreted during a dwarf nova outburst (obtained by measuring the total energy radiated, $\sim 10^{39}-10^{40} \mathrm{erg}$, and ascribing it to released gravitational energy) is $\sim 10^{22} \mathrm{~g}$ (cf. $10^{33}$ $\mathrm{g}$ for the whole primary) and can thus be easily tugged around by magnetic coupling to the accretion disc. It spins up as $\dot{M}$ increases on the rising branch of outburst, which compresses the magnetosphere of the primary and decreases the period at the inner radius of the disc. Because $\dot{M}$ varies with time during an outburst, no equilibrium is reached. As the inner radius of the disc steadily decreases, the minimum energy state for accretion is constantly being sought, so field lines reconnect between the equatorial belt (which has differential rotation in latitude as well as in depth) and the disc, resulting in accretion along field lines connected to zones of slightly different periods of rotation. This search for equilibrium causes the sudden jumps of DNO period as matter is transferred from one magnetic channel to another; they are least frequent at maximum luminosity when $\dot{M}$ passes through its turning point and hence is most constant.

After maximum luminosity, $\dot{M}$ decreases steadily, and at some point the inner edge of the disc retreats so rapidly that the belt is not able to slow its rotation period rapidly enough to maintain near equilibrium. Then gas attaching itself to the field lines anchored in the belt is centrifuged outwards, stopping most of the accretion onto the primary and extracting angular momentum from the belt.

Evidence for such 'propellering' is seen in VW Hyi. The variation in DNO period as a function of brightness on the descending branch of outburst is shown in Fig. 10. The general correlation between period and brightness for $8.3<V<12.3$ is an example of the 
ubiquitous period-luminosity relationship referred to above. But that is succeeded by a phase of extremely rapid increase in period, during which the DNO period doubles in about 6 hours. This phase is concomitant with cessation of EUV flux (Fig. 11). As EUV flux is a competent monitor of $\dot{M}$ onto the primary, it can be seen that accretion is reduced to a trickle exactly at the time that the deduced rapid deceleration of the equatorial belt is occurring.

What about lpDNOs? Their independence from accretion luminosity puts them in a different category from ordinary DNOs, but in other aspects they behave like magnetically channelled accretion, including the existence of double lpDNOs split at the QPO frequency (Section 4.1). A clue to the cause of their longer periods comes from observed rotationally broadened spectra of some CV primaries. Taking the observed projected rotational velocities (Sion 1999) and adopting measured or estimated masses and inclinations (details are given in WW2b and WWP) we find the following rotation periods (all with at least 20\% uncertainty): VW Hyi: 140 s; SS Cyg: 63 s; OY Car: >260 s. These rotation periods are roughly twice the observed lpDNO periods, which would occur for accretion onto two regions of the primary. I suggest, therefore, that lpDNOs are literally connected to the primary's rotation, which will be differential in latitude as material accreted at the equator spreads out across the surface. This explains why, unlike DNOs, lpDNO periods are not correlated with $\dot{M}$ and they behave independently of DNOs.

If this interpretation is correct then the lpDNOs allow an extension of knowledge of rotation periods - from the IP region to primaries of lower field strength. The definite lpDNOs in Table 1 cover the range 33 - 177 s. If we exclude AE Aqr, then the presently known IPs have rotation periods that range from $142 \mathrm{~s}$ of DQ Her to $4021 \mathrm{~s}$ for EX Hya. The reason for omitting AE Aqr is because the field of $\sim 3 \times 10^{5} \mathrm{G}$ deduced for it (Wynn, King \& Horne 1997; Choi \& Yi 2000) is at the bottom end of the range of IP fields and may 
not be quite large enough for the primary to behave as a solid body. This in turn calls into question the high spin-down power that is deduced from the observed large $d P_{\text {rot }} / d t$ in $\mathrm{AE}$ Aqr (de Jager et al. 1994): indeed, the rapid spin-down may be simply because only the inertia of the outer layers of the primary is coupled to the retarding torque (which, because of the high rotation rate, is largely due to propellering).

The existence of two clear groups - rapidly rotating primaries with signatures of weak fields, and slower rotating IPs with quite strong fields - constitutes a correlation between magnetic field strength and rotation period of the primary. This is related to the fact that the stronger the field, the larger is the radius of the magnetosphere, and the longer is the IP equilibrium period. But $\dot{M}$ also plays a role - the larger the $\dot{M}$, the smaller the equilibrium radius of the magnetosphere. And from the observed time scales $\frac{P_{\text {rot }}}{\left|d P_{\text {rot }} / d t\right|} \sim 10^{6}$ y in IPs we see that it is the average $\dot{M}$ over the past millions of years that determines the current value of Prot. Such a time scale probably includes several nova explosions, and may include long periods of very low $\dot{M}$ (hibernation - see Section 9.4.3 of Warner 1995a), so the apparent correlation between field strength and spin period means either that the presently weak field CVs have preferentially had historic high $\dot{M}$, or that all CVs experience roughly similar average $\dot{M}$ and that their spin periods are solely a result of their weaker fields. The latter scenario seems more probable, and, indeed, if measurements of $B$ can be made, offers a way to estimate long-term average values of $\dot{M}$.

I have suggested that DNOs and lpDNOs are the signatures of magnetically channelled accretion onto regions that are rotating respectively with maximum angular velocities close to the equatorial Keplerian velocity and at roughly one eighth (for two-pole accretion) of that velocity. According to Kippenhahn \& Thomas (1978), the large specific angular momentum of material accreted at the equator onto a non-magnetic primary results in very slow mixing to higher latitudes: the time scale is much greater than the time between 
nova eruptions (but note that this computation includes only mixing via the Richardson instability; coupling of parts of the surface by closed loop magnetic fields will also distribute angular momentum). They find that the latitudinal width of the equatorial belt, caused by mixing and defined as that latitude where the velocity falls to half of the equatorial velocity, is $\sim 20^{\circ}$ (beyond which it falls very rapidly). They also find that $10^{4} \mathrm{y}$ of accretion at $10^{-9}$ $\mathrm{M}_{\odot} \mathrm{y}^{-1}$ results, after mixing with surface material, in a belt with an angular velocity 0.22 of the Keplerian velocity in the equatorial plane - and therefore $\sim 0.11$ at latitude $20^{\circ}$. In the suggested model for lpDNOs this would mean that the body of the primary (or, at least where accretion onto it occurs) has acquired a rotation period close to that of the belt at its outer edge.

Perhaps the most direct evidence so far for magnetically controlled accretion in a dwarf nova comes from the analysis of XMM-Newton observations of OY Car just after an outburst. Wheatley \& West (2003) show that the eclipse implies that the X-Ray emitting region is noticeably smaller than the surface of the primary, and is away from the equator (at latitude $\sim 50^{\circ}$ in their diagram). It is the presence of such accretion that may account for the rare $48 \mathrm{~s}$ QPOs observed in OY Car during quiescence (WWP) and the $60 \mathrm{~s}$ X-Ray QPOs seen in VW Hyi in quiescence (Pandel, Cordova \& Howell 2003).

Almost as direct evidence is given by the presence of a variable strength inverse $\mathrm{P}$ Cygni feature observed in the spectrum of VW Hyi during outburst, which has been interpreted as structured inflow of gas from the inner edge of a truncated disc (Huang et al. 1996). 


\section{THE NATURE OF QPOs}

The observational evidence available at present shows (WW2b) that when a double DNO occurs then it is the shorter of the two periods that is at the expected DNO period - and the profile of that signal is sinusoidal. The longer period often has a significant first harmonic (e.g., Marsh \& Horne 1998; Fig. 12). The fact that the DNO frequency is accompanied by only one sideband shows that this is not a case of modulation of the $\mathrm{DNO}$ at the QPO frequency; rather it is similar to the dominant sideband seen in IPS which is caused by 'reflection' (more correctly, reprocessing) of the rotating beam off the secondary (or other structure, such as a thickening of the disc at stream impact, revolving at the orbital frequency). By analogy we conclude that the longer period DNO component is generated by the rotating DNO beam being reprocessed by a structure revolving in a prograde direction around the primary at the QPO period. The irregular profile of this structure causes the harmonics in the reprocessed signal.

The periods of most QPOs are so short (relative to the orbital period) that the accretion disc is the only possible location for the reprocessing site. If revolving at the Keplerian period the site would be in the outer region of the disc and would have to have a very large vertical height in order to intercept enough of the rotating DNO beam. This is not out of the question, but perturbation analyses of accretion discs show that there are a number of oscillatory modes available, and that a slowly moving prograde travelling wave in the inner disc is the most likely to be excited (Lubow \& Pringle 1993). Such a travelling wave, excited by a process of magnetic winding and reconnection, has been proposed as the structure that generates the double DNOs (WW2b). The QPOs themselves are then the result of reprocessing and/or obscuration of the radiation from the hot inner regions by the travelling wave.

The obscuration aspect of QPOs is demonstrated by the occasional presence of deep 
dips at the minima of the QPO modulations, which pull the light curve well below its interpolated lower envelope (see, e.g., Figure 2 of WW2b). A particularly interesting QPO profile is shown by SW UMa, in which the minimum of the $\sim 370$ s modulation is deepened by an apparent shallow eclipse of $\sim 70 \mathrm{~s}$ duration, which is probably a partial eclipse of the primary (Fig. 14).

This model for optical QPOs - simple reflection and obscuration by an excited mode of the accretion disc - differs greatly from some earlier proposals that also invoke oscillation modes of discs. In those (non-magnetic) studies (e.g. Carroll et al. 1985; Collins, Helfer \& van Horn 2000) the QPOs are supposed to be oscillations of the intrinsic luminosity of the disc itself. This is a viable option for non-magnetic primaries, but it remains to be demonstrated observationally that such oscillation modes are excited and generate detectable QPOs.

The QPOs observed in the hard X-Ray emission of SS Cyg during outburst are not entirely compatible with the above model. Although deep dips that appear to be obscurations accompany the QPOs (Figure 9 of WMM), the quasi-periodic peaks cannot be due to a reflection effect. The overall effect looks more like QPOs of $\dot{M}$ at the inner boundary layer.

In this regard it is of interest to note parallel studies in other fields of astrophysics that have relevance to the QPOs in CVs. Quasi-periodic accretion and ejection of dense knots of gas in young stellar objects (YSOs) has been modelled as an interaction between a magnetic star and its accretion disc. The inner boundary of the disc undergoes quasi-periodic radial oscillations, each of which strips a ring of gas that falls onto the star (Goodson, Böhm \& Winglee 1999; Goodson \& Winglee 1999). In the application to YSOs, appropriate parameters lead to an estimate of the oscillation period as $\sim 100 / 2 \pi$ times the Keplerian period at the inner edge of the disc. It can be shown that the same mechanism applied 
to high $\dot{M} \mathrm{CV}$ discs and $B \sim 10^{6} \mathrm{G}$ produces a similar result, which we can interpret as $P_{Q P O} / P_{D N O} \sim 100 / 2 \pi \sim 16$. Such modulation of accretion onto low field CV primaries is therefore another mechanism that could produce QPOs at the observed periods. These would probably look more like flares than sinusoidal modulations. A possible example is shown in Fig. 13 where recurrent flares with a quasi-period of $\sim 750 \mathrm{~s}$ are seen. In this light curve, low amplitude ordinary QPOs at $347 \mathrm{~s}$ were also present, demonstrating the permitted co-existence of the two types of QPO.

In similar modelling by Uzdensky (2002) and Uzdensky, Königl \& Litwin (2002) of accretion from a disc into the magnetosphere of an aligned magnetic rotator, it is found that field winding and reconnection can lead to quasi-periodic accretion. The first results of 3D modelling of accretion onto an inclined dipole rotator have recently appeared in which short time scale QPOs also are found (Romanova et al. 2003).

\section{FREQUENCY DOUBLING AND ALIASING}

DNOs in the EUV region of SS Cyg have been observed in several outbursts (Mauche 1996b, 1997b, 1998, 2002) and constitute the most complete coverage of the evolution of DNOs in this wavelength region. The DNOs were found to be mostly sinusoidal in profile, except that at the brightest parts of outbursts in August 1993 and June 1994 a noticeable first harmonic appeared. Then, in the October 1996 outburst, with SS Cyg at its brightest, the fundamental disappeared and the signal doubled in frequency to become entirely first harmonic, i.e. with a period $\sim 3 \mathrm{~s}$. The next outburst was observed in soft X-Rays and was also found to have a $2.8 \mathrm{~s}$ DNO (van Teeseling 1997).

Before discussing possible physical causes of the frequency doubling, it is useful to point out that such short oscillation periods have implications for optical photometry of 
DNOs. For example, the 9.735 s DNO observed in SS Cyg by Patterson, Robinson and Kiplinger (1978) at the unprecedentedly low amplitude of 0.02 mmag is now seen (as was allowed by the authors at the time) to be a beat between a true period of $6.790 \mathrm{~s}$ (or its first harmonic) and the photometric integration time of 4s. If under-sampled in such a way, a sinusoidal signal is reduced in amplitude by a factor of $\sin x / x$, where $x=p d t / P_{D N O}$ and $d t$ is the integration time. For the case under consideration, the true amplitude of the $6.79 \mathrm{~s}$ DNO would therefore have been $0.04 \mathrm{mmag}$, or $0.14 \mathrm{mmag}$ if frequency doubling had occurred.

Another interesting example is given by the 'type star' for QPOs - namely, RU Peg. The Fourier transform for RU Peg computed by Patterson, Robinson \& Nather (1977) shows a QPO centred on $50 \mathrm{~s}$ and a $0.6 \mathrm{mmag}$ DNO at $11 \mathrm{~s}$; their integration time was 4s. From the discussion in Section 4 we would expect DNOs at $\sim 50 / 15 \sim 3.3$.s, not at $11 \mathrm{~s}$. One possibility, therefore, is that the observed signal at $11 \mathrm{~s}$ was a beat with the integration time, and that the true signal was $2.97 \mathrm{~s}$ with an amplitude of 2.8 mmag. This opens the further possibility that RU Peg had undergone frequency doubling at that time. Yet another possibility is that the observed $11 \mathrm{~s}$ signal was a low amplitude lpDNO and there was no ordinary DNO detected at all.

The lesson to be learnt from this is that it is necessary either to carry out photometry of DNOs with integration times of $1 \mathrm{~s}$ or less, or alternatively to split up the observations using non- commensurate integration times - e.g. alternate runs with $4 \mathrm{~s}$ and $5 \mathrm{~s}$ integrations.

The physical cause of frequency doubling is not yet clear - it is a topic to be explored that could lead to greater insight into normal DNO behaviour. A transition from single pole to two-pole accretion is an obvious possibility. As $\dot{M}$ increases to a maximum value the magnetosphere is squashed close to the surface of the white dwarf, at which point higher multipole components of the magnetic field geometry become more resilient than the dipole 
component (Lamb 1988) and what may have been a single visible accretion region could become two or more accreting zones (though only one or two are likely to capture most of the accretion flow).

Another possible model results from the change in viewing geometry when the inner radius of the disc approaches the surface of the primary. At moderate to high orbital inclinations the primary obscures the inner part of the rear of the disc, and the front side of the disc, with its accretion curtain, can obscure the lower hemisphere and perhaps even part of the equatorial accretion zone. Furthermore, accretion columns or curtains are often of lowest optical thickness perpendicular to the accretion flow, which produces a fan beam. In either case the maximum direct visibility of an accretion zone may be near each limb of the rotating primary with the result that a single bright region will be seen twice per rotation.

It would be helpful to have simultaneous EUV/X-Ray and optical observations - the different wavelength regions give different points of view, the former showing what happens as seen directly from the centre of the system, and the latter showing largely what the disc sees. It is possible that frequency doubling could occur at the short wavelengths but not at visible wavelengths.

An instructive example of a powerful effect of geometry is given by the IP XY Ari, in which the amplitude (in X-Rays) of the $206 \mathrm{~s}$ periodic pulse is $\sim 20 \%$ and is double peaked in quiescence but $\sim 90 \%$ and single peaked in outburst. This is an example of period doubling rather than frequency doubling during outburst, the interpretation of which is that in quiescence there are two almost equal and $180^{\circ}$ out of phase pulses, coming from two-pole accretion, but during an outburst the inner edge of the disc moves so close to the primary that the lower pole is obscured, removing the filling-in effect of the other pole (Hellier, Mukai \& Beardmore 1997).

But there may be other reasons for frequency doubling, especially when we note that 
QPOs can also double (or halve) their frequencies. The QPOs in VW Hyi appear to double in frequency (WW2b) when the system is approaching quiescence, when the inner edge of the disc must be well above the surface of the primary and cannot be responsible for any geometric effects. Frequency doubling of QPOs in KT Per has been reported (Robinson \& Nather 1979). The claimed steady decrease in period of QPOs in EF Peg (Kato 2002) is probably more realistically interpreted as an approximate frequency doubling near the middle of the train of oscillations. These may be understood as switches to and from predominantly fundamental and first harmonic excitations of the QPO travelling wave, but the cause for this remains unknown.

\section{WZ Sge}

WZ Sge is of particular interest because it seems to be an IP while in quiescence and a DNO machine when in outburst - a result of the primary having a magnetic moment almost strong enough to anchor the exterior to the interior. I use 'almost' because in quiescence

prior to the 1978 outburst, $P / \dot{P} \sim 1 \times 10^{5}$ y (see Figure 7 of Patterson 1980), which is too short a time scale to be a spin- down of the entire primary.

\subsubsection{WZ Sge in Quiescence}

The connection between WZ Sge and DNO/QPO phenomena has taken a long time to demonstrate convincingly. Patterson et al. (1998) reviewed the optical observations and added ASCA hard X-Ray observations showing modulation at $27.87 \mathrm{~s}$, which are characteristic of magnetic channelling onto a white dwarf. HST observations also show the dominance of $27.87 \mathrm{~s}$ (Skidmore et al. 1999). The observed $v \sin i$ (Cheng et al. 1997) for the primary of WZ Sge leads to a rotation period $28 \pm 8 \mathrm{~s}$, which is in agreement with the 
magnetic accretor model.

The presence of an additional persistent 28.952 s modulation, which bears no simple relationship to that at $27.87 \mathrm{~s}$, led some workers to suggest that it may be a non-radial oscillation of the primary (e.g. Robinson, Nather \& Patterson 1978). Patterson (1980) originally pointed out that the $28.95 \mathrm{~s}$ period could be due to reprocessing of a $27.87 \mathrm{~s}$ rotating beam from a progradely-moving thickening of the disc. Lasota, Kuulkers \& Charles (1999) suggested that this reprocessing site could be at the outer rim of the accretion disc, moving at the Keplerian period, but WW2b suggested, in analogy with their findings in outbursting dwarf novae, that the site is near the inner edge of the disc. The existence of the hypothesised disc thickening, moving with a revolution period of $744 \mathrm{~s}$ (the beat period between the two short periods), has in fact been directly demonstrated (WW2b). FTs of some of the light curves show that there is a $744 \mathrm{~s}$ period present (it is probably there at all times, but the large variation of amplitude from one cycle to the next makes it difficult to detect in the FT) - in the light curve it appears as recurrent dips of variable depth, the most prominent of which is around orbital phase 0.25 and has been seen in almost all optical curves from the earliest observations (Krzeminski \& Kraft 1964). The obscuring source produces the deepest dip when it transits across the bright spot as seen from behind; it does not transit the bright spot when seen from the front, so there is no corresponding deep dip near phase 0.75 .

The infrared (K-band) light curve of WZ Sge is far less variable than the light curves at shorter wavelengths and is largely due to the modulation caused by viewing the bright spot through an optically thin disc (Skidmore et al. 2002). Interestingly, no QPO dips are seen in the IR (Ciardi et al. 1998; Skidmore et al. 2002), so we deduce that the travelling wave responsible for the QPO dip obscures only the hotter central region of the bright spot, and not the extended cooler region. 
To be consistent with the interpretation used earlier, we should classify the $27.87 \mathrm{~s}$ modulation as an lpDNO, related to rotation of the main body of the primary, not a DNO. The 27.87/28.95 s pair constitutes a double lpDNO. More specifically, WZ Sge is an IP of the DQ Her subclass (Warner 1995a), but is of lower Q than typical IPs.

\subsubsection{WZ Sge in Outburst}

The 2001 superoutburst of WZ Sge provided an opportunity to observe how the short period oscillations behave during times of greatly increased $\dot{M}$. DNOs were detected only one month after the peak of outburst (Knigge et al. 2002; hereafter K2002), and later from two to five months after peak brightness (Welsh et al. 2003; hereafter W2003). As pointed out by the latter authors, the temperature of the primary fell from $29000 \mathrm{~K}$ to $18000 \mathrm{~K}$ during that time, yet the modulations did not change their character appreciably - this is a characteristic of DNOs rather than white dwarf pulsations.

The earliest observed oscillations (K2002) have periods near 15 s and show changes in amplitude and phase that are characteristic of DNO behaviour. There were also weak oscillations with periods near $6.5 \mathrm{~s}$. In the later observations (W2003) the $28.96 \mathrm{~s}$ oscillation was present with some stability, but not the $27.87 \mathrm{~s}$ one. In addition, there was an $18 \mathrm{~s}$ oscillation of lower coherence, characteristic of a DNO.

If we allow that the magnetic field of WZ Sge is barely strong enough to couple the white dwarf's exterior to its interior (i.e. $B \sim 5 \times 10^{5} \mathrm{G}$ ) then we would expect that the very large $\dot{M}$ during superoutburst would squash the magnetosphere to the surface of the primary. But field enhancement in the equatorial belt could allow at least some of the accretion to be magnetically channelled. The equatorial belt would start with the spin period of the primary itself, i.e. $27.87 \mathrm{~s}$, and would be spun up from there - 
perhaps producing the $15 \mathrm{~s}$ and $18 \mathrm{~s}$ DNOs, which appear correlated in the usual way with luminosity of the system. For lpDNOs of $28 \mathrm{~s}$ we would expect, from the relationships found for other dwarf novae (Section 3), DNOs at about one quarter of that period - i.e. 6 $\mathrm{s}$, which is close to the observed $6.5 \mathrm{~s}$ DNOs.

Therefore, phenomenologically, WZ Sge behaves in outburst in ways similar to other weakly magnetic dwarf novae. Nevertheless, the appearance of the reprocessed signal at 28.96 s so early in the outburst decline, which implies the existence of the 744 s travelling wave in the model outlines above, requires its excitation process and period to be amazingly robust - and apparently independent of the $\dot{M}$ passing through the inner disc.

\section{A CONNECTION WITH X-RAY BINARIES}

Many of the phenomena exhibited by CVs also appear in X-Ray binaries (XRBs), which have neutron stars and black hole $(\mathrm{BH})$ candidates accreting from companions. Rapid modulations are among the common properties, though in the XRBs the signals appear at hard X-Ray energies where the flux is low and until recently the oscillations were only detectable in Fourier transforms of many thousands of cycles. An overview of QPOs in XRBs is given by van der Klis (2002).

Some specific similarities to $\mathrm{CV}$ behaviour are seen in the phase variations in the $5 \mathrm{~Hz}$ QPOs in the Rapid Burster (Dotani et al. 1990), the double QPOs at kHz frequencies, and the frequency dependence on accretion luminosity. But a striking result is that the ratio of low to high frequency QPOs that appear in the XRBs is close to the value 15 seen in CVs. By considering the CV QPOs and DNOs as low and high frequency QPOs we can place them on the same diagram as for XRBs - Fig. 15. The correlation is seen to extend over nearly six orders of magnitude in frequency. This does not necessarily mean that exactly 
the same physics is in operation over the entire range, but certainly the same ratio of time scales appears everywhere.

Furthermore, during X-Ray bursts oscillations are often seen that lie between the high and low frequency QPOs. They vary in frequency and are thought to be caused by hot expanding gas on the surface of the neutron star that slips relative to the underlying surface (van der Klis 2000). They are closely related to the rotation period of the star itself and thus resemble the lpDNOs in CVs described in this review.

A recent important development in the study of X-Ray Binary QPOs is an analysis of the signal in the neutron star system 4U1608-52 (Barret, Olive \& Kluzniak 2003) in which much higher time resolution has been achieved. What had been thought to be high frequency $(800 \mathrm{~Hz})$ QPOs with $Q \sim 10$ have now been shown to be short (hundreds of cycles) trains of higher ( $Q$ up to 103) coherence with a duty cycle $\sim 15 \%$ and jumps in frequency of up to $\sim 0.5 \%$ between the bursts of QPOs. Apart from the implication that the QPO amplitudes are typically $\sim 8$ times what had previously been deduced, the high stability for so many cycles eliminates many models of X-Ray QPOs (e.g., blobs of accreting gas that would be sheared out of existence after only $\sim 10$ cycles). During the phases of high stability the average profile of the QPO is closely sinusoidal.

Consequently the high frequency X-Ray QPOs now look even more like CV DNOs with period jumps after $\sim 100$ cycles. Among the CVs there are examples of DNOs that are present for only part of the time (i.e., a short duty cycle), but also ones (e.g. VW Hyi just before quiescence) where high amplitude is maintained for at least thousands of cycles.

The similarities between $\mathrm{CV}$, neutron star and $\mathrm{BH}$ rapid oscillations may have profound implications. Robertson \& Leiter (2003), in gathering evidence that some BHs may have magnetic moments, cite these similarities in their compilation. They reason that BHs forming from magnetic stars are prevented within a Hubble time from reaching their event 
horizons by the radiation pressure that results from pair-creation in the greatly compressed and intensified internal magnetic fields. If this is the case, then magnetic stars collapsing towards the $\mathrm{BH}$ end point behave temporarily like over-massive neutron stars, and have more in common with magnetic neutron stars and white dwarfs than has been previously realised.

\section{ACKNOWLEDGEMENTS}

I am greatly indebted to my co-workers in this field - Dr Patrick Woudt, Claire Blackman and Retha Pretorius - for communicating results in advance of publication, and for assistance. Our observations have largely been obtained at the Sutherland station of the South African Astronomical Observatory. I thank Steve Potter for helpful comments. My research is supported by funds from the University of Cape Town. I acknowledge the

hospitality of the Nicolaus Copernicus Center in Warsaw, where part of this Review was written. 


\section{REFERENCES}

Araujo-Betancor, S. 2003, IAU Coll. 190, ASP Conf. Ser, in press

Bailey, J. 1980, MNRAS, 190, 119

Barret, D., Olive, J.-F., \& Kluzniak, W. 2003, Nature, submitted

Bruch, A., Steiner, J.E., \& Gneiding, C.D. 2002, PASP, 112, 237

Carroll, B.W., Cabot, W., McDermott, P.N., Savedoff, M.P., \& van Horn, H.M. 1985, ApJ, 296,529

Chanan, G.A., Nelson, J.E., \& Margon, B. 1978, ApJ, 226, 963

Chen, A., O’Donoghue, D., Stobie, R.S., Kilkenny, D., \& Warner, B. 2001, MNRAS, 325, 89

Cheng, F.H., Sion, E.M., Horne, K., Hubeny, I., Huang, M., \& Vrtilek, S.D. 1997, AJ, 114, 1165

Choi, C.-S., \& Yi, I. 2000, ApJ, 538, 862

Ciardi, D., Howell, S., Hauschildt, P., \& Allard, F. 1998, ApJ, 504, 450

Collins, T.J.B., Helfer, H.L., \& van Horn, H.M. 2000, ApJ, 534, 944

Cordova, F.A., \& Mason, K.O. 1984, MNRAS, 206, 879

Cordova, F.A., Chester, T.J., Tuohy, I., \& Garmire, G.P. 1980, ApJ, 235, 609

Cordova, F.A., Chester, T.J., Mason, K.O., Kahn, S.M., \& Garmire, G.P. 1984, ApJ, 278, 739

de Jager, O.C., Meintjes, P.J., O’Donoghue, D., \& Robinson, E.L. 1994, MNRAS, 267, 577

Durisen, R.H. 1973,ApJ, 183, 215 
Eracleous, M., Patterson, J., \& Halpern, J.P. 1991, ApJ, 370, 330

Gänsicke, B.T., \& Beuermann, K. 1996, A\&A, 309, L47

Goodson, A.P., \& Winglee, R.M. 1999, ApJ, 524, 159

Goodson, A.P., Böhm, K.-H., \& Winglee, R.M. 1999, ApJ, 524, 142

Hassall, B.J.M., Pringle. J.E., Schwarzenberg-Czerny, A., Wade, R., Whelan, J.A.J., \& Hill, P.W. 1983, MNRAS, 203, 865

Haefner, R., Schoembs, R., \& Vogt, N. 1979, A\&A, 77,7

Hesser, J.E., Lasker, B.M., \& Osmer, P.S. 1974, ApJ, 189, 315

Hellier, C., Mukai, K., \& Beardmore, A.P. 1997, MNRAS, 292, 397

Hildebrand, R.H., Spillar, E.J., \& Stiening, R.F. 1981, ApJ, 248, 268

Huang, M., Sion, E.M., Hubeney, I. Cheng, F.H., \& Szkody, P. 1996, ApJ, 458, 355

Ishida, M., Yamashita, A., Ozawa, H., Nagase, F., \& Inoue, H. 1996, IAU Circ. 6340

Jensen, K.A., et al. 1983, ApJ, 270, 211

Jones, M.H., \& Watson, M.G. 1992, MNRAS, 257, 633

Kato, T. 2002, PASJ, 54, 87

Kato, T., \& Starkey, D.R. 2002, IBVS No. 5358

Kato, T., Hirata, R., \& Mineshige, S. 1992, PASJ, 44, 215L

Kato, T., Ishioka, R., \& Makoto, U. 2002, PASJ, 54, 1033

Kato, T., et al. 2003, PASJ, 55, 489 
Kato. T., et al. 2004, MNRAS, in press

Kippenhahn, R., \& Thomas, H.-C. 1978, A\&A, 63, 265

Knigge, C., Drake, N., Long, K.S., Wade, R.A., Horne, K., \& Baptista, R. 1998, ApJ, 499, 429

Knigge, C., Hynes, R.I., Steeghs, D., Long, K.S., Araujo-Betancor, S., \& Marsh, T.R. 2002, ApJ, 580, 151 (K2002)

Kraicheva, Z., Stanishev, V., \& Genkov, V. 1999a, A\&AS, 134, 263

Kraicheva, Z., Stanishev, V., Genkov, V., \& Illiev, L. 1999b, A\&A, 351, 607

Krzeminski, W., \& Kraft, R.P. 1964, ApJ, 140, 921

Kuulkers, E., Norton, A., Schwope, A., \& Warner, B. 2003, in Compact Stellar X-Ray Sources, eds., W.H.G. Lewin \& M. van der Klis (Cambridge University Press), in press

Lamb, D.Q. 1988, in Polarized Radiation of Circumstellar Origin, eds., G.V. Coyne et al., Vatican Obs., Vatican, p.151

Larsson, S. 1992, A\&A, 265, 133

Lasota, J.-P., Kuulkers, E., \& Charles, P.A. 1999, MNRAS, 305, 473

Liebert, J., Bergeron, P., \& Holberg, J.B. 2003, AJ, 125, 348

Long, K.S., Mauche, C.W. et al. 1996, ApJ, 469, 841

Lubow, S.H., \& Pringle J.E. 1993, ApJ, 409, 360

Marsh, T.R., \& Horne, K. 1998, MNRAS, 299, 921 
Martell, P.J., Horne, K., Price, C.M., \& Gomer, R.H. 1995, ApJ, 448, 380

Mason, K.O., Cordova, F.A., Watson, M.G., \& King, A.R. 1988, MNRAS232, 779

Mauche, C.W. 1996a, ApJ, 463, L87

Mauche, C.W. 1996b, in Astrophysics of the Extreme Ultraviolet, eds., S. Bowyer \& R.F. Malina (Kluwer: Dordrecht), 317

Mauche, C.W. 1997a, ApJ, 476, 85

Mauche, C.W. 1997b, ASP Conf. Ser. 121, 251

Mauche, C.W. 1998, ASP Conf. Ser. 137, 113

Mauche, C.W. 2002, ApJ, 580, 423

Mauche, C.W., \& Robinson, E.L. 2001, ApJ, 562, 508

Morales-Rueda, L., Still, M.D., \& Roche, P. 1996, MNRAS, 283, 58

Morales-Rueda, L., Still, M.D., \& Roche, P. 1999, MNRAS, 306, 753

Nather, R.E., \& Robinson, E.L. 1974, ApJ, 190, 637

Nogami, D., Kato, T., \& Baba, H. 2002, PASJ, 54, 987

Nogami, D., Baba, H., Kato, T., \& Novak, R. 1998, PASJ, 50, 297

O'Donoghue, D. 1995, Balt.Astr. 4, 517

O’Donoghue, D., Menzies, J.M., \& Hill, P.W. 1987, MNRAS, 227, 347

Paczynski, B. 1978, in Nonstationary Evolution of Close Binaries, ed. A. Zytkov (Polish Sci. Publ.: Warsaw), 89 
Pandel, D., Cordova, F., \& Howell, S.B. 2003, MNRAS, in press

Patterson. J. 1979, ApJ, 233, L13

Patterson, J. 1981, ApJS, 45, 517

Patterson, J., Robinson, E.L., \& Kiplinger, A.L. 1978, ApJ, 226, L137

Patterson, J., Robinson, E.L., \& Nather, R.E. 1977, ApJ, 214, 144

Patterson, J., Robinson, E.L., \& Nather, R.E. 1978, ApJ, 224, 570

Patterson. J., Nather R.E., Robinson, E.L., \& Handler, F. 1979, ApJ, 232, 819

Patterson, J., Sterner, E., Halpern, J.P., \& Raymond, J.C. 1992, ApJ, 384, 234

Patterson, J., Jablonski F., Koen, C., O’Donoghue, D., \& Skillman, D.R. 1995, PASP, 107, 1183

Patterson, J., Richman, H., Kemp, J., \& Mukai, K. 1998, PASP, 110, 403

Patterson, J., Thorstensen, J.R., Fried, R.E., Skillman, D.R., Cook, L.M., \& Jensen, L. 2001, PASP, 113, 72

Patterson, J., et al. 2002a, PASP, 114, 721

Patterson, J., et al. 2002b, PASP, 114, 1364

Patterson, J., et al. 2002c, PASP, 114, 65

Pavlenko, E.P., \& Shugarov, S.Yu. 1999, A\&A, 343, 909

Petterson, J. 1980, ApJ, 241, 247

Provencal, J.L., \& Nather, R.E. 1997, ApSpSci Libr 214, 67 
Provencal, J.L., et al. 1995, ApJ, 445, 927

Ramsay, G. et al. 2001, A\&A, 365, 288

Regos, E., \& Tout, C.A. 1995, MNRAS, 273, 146

Remillard, R.A., et al. 1994, ApJ, 428, 785

Robertson, S.L., \& Leiter, D.J. 2003, ApJ, in press

Robinson, E.L., \& Nather, R.E. 1979, ApJS, 39, 461

Rodriguez-Gil, P., \& Martinez-Pais, I.G. 2002, MNRAS, 337, 209

Rodriguez-Gil, P., Casares, J., Martinez-Pais, I.G., Hakala, P., \& Steeghs, D. 2001, ApJ, 548, L49

Rodriguez-Gil, P., Casares, J., Martinez-Pais, I.G., \& Hakala, P. 2002, ASP Conf. Ser. 261, 533

Romanova, M.M., Ustyugova, G.V., Koldoba, A.V., Wick, J.V., \& Lovelace, R.V.E. 2003, ApJ, 595, 1009

Silber, A.D., Anderson, S.F., Margon. B., \& Downes, R.A. 1996, AJ, 112, 1174

Sion, E.M. 1999, PASP, 111, 532

Sion, E.M., \& Urban, J. 2002, ApJ, 572, 456

Sion, E.M., Cheng, F.H., Huang, M., Hubeny, I., \& Szkody, P. 1996, ApJ, 471, L41

Skidmore, W., Welsh, W.F., Wood, J.H., \& Stiening, R.F. 1997, MNRAS, 288, 189

Skidmore, W., Welsh, W.F., Wood, J.H., Catalán, M.S., \& Horne, K. 1999, MNRAS, 310, 750 
Skidmore, W., Wynn, G.A., Leach, R., \& Jameson, R.F. 2002, MNRAS336, 1223

Skillman, D.R., Patterson, J., \& Thorstensen, J.R. 1995, PASP, 107, 545

Skillman, D.R., et al. 1999, PASP, 111, 1281

Smith, D.A., Dhillon, V.S., \& Marsh. T.R. 1998, MNRAS, 296, 465

Steeghs, D., O’Brien, K., Horne, K., Gomer, R., \& Oke, J.B. 2001, MNRAS, 323, 484

Szkody, P., Hoard, D.W., Sion, E.M., Howell, S.B., Cheng, F.H., \& Sparks, W.M. 1998, ApJ, 497, 928

Szkody, P., Nishikida, K., Long, K.S., \& Fried, R. 2001, AJ, 121, 2761

Taylor, C.J., Thorstensen, J.R., \& Patterson, J. 1999, PASP, 111, 184

Tovmassian, G.G. 1988, Adv. Sp. Res. 8, 329

Tremko, J., et al. 1996, A\&A, 312, 121

Uemura, M., et al. 2001, VSNET-Campaign 786

Uzdensky, D.A. 2002, ApJ, 572, 432

Uzdensky, D.A., Königl, A., \& Litwin, C. 2002, ApJ, 565, 1205

van der Klis, M. 2000, ARA\&A, 38, 717

van der Woerd, H., Heise, J., \& Bateson, F. 1986, A\&A, 156, 252

van Teeseling, A. 1997, A\&A, 324, 73

Walker, M.F. 1956, ApJ, 123, 68

Warner, B. 1988, High Speed Photometry (Cambridge University Press) 
Warner, B. 1995a, Cataclysmic Variable Stars (Cambridge University Press)

Warner, B. 1995b, ASP Conf. Ser. 85, 343

Warner, B., \& Brickhill, A.J. 1978, MNRAS182, 777

Warner, B., \& Robinson, E.L. 1972, Nature Phys. Sci., 239, 2

Warner, B., \& Woudt, P.A. 2002, MNRAS, 335, 84 (WW2b)

Warner, B., O’Donoghue, D., \& Allen, S. 1985, MNRAS, 212, 9P

Warner, B., O'Donoghue, D., \& Wargau, W. 1989, MNRAS, 238, 73

Warner, B., Woudt, P.A., \& Pretorius, M.L. 2003, MNRAS, 344, 1193 (WWP)

Warner, B., Peters, W.L., Hubbard, W.B., \& Nather, R.E. 1972, MNRAS159, 321

Watson, M.G., King, A.R., \& Heise, J. 1985, Sp. Sci. Rev., 40, 127

Watson, M.G., King, A.R., \& Osborne, J. 1995, MNRAS, 212, 917

Welsh, W.F., Skidmore, W., Wood, J.H., Cheng, F.H., \& Sion, E.M. 1997, MNRAS, 291, 57

Welsh, W.F., Sion, E.M., Godon, P., Gänsicke, B.T., Knigge, C., Long, K.S., \& Szkody, P. 2003, ApJ, in press (W2003)

Wheatley, P.J., \& West, R.G. 2003, MNRAS, 345, 1009

Wheatley, P.J., et al. 1996, A\&A, 307, 137

Wheatley, P.J., Mauche, C.W., Mattei, J.A. 2003, MNRAS, 345, 49

Wickramasinghe, D.T., \& Ferrario, L. 2000, PASP, 112, 873

Williams, G.A., \& Hiltner, W.A. 1984, MNRAS, 211, 629 
Woudt, P.A., \& Warner, B. 2002a, MNRAS, 333, 411 (WW2a)

Woudt, P.A., \& Warner, B. 2002b, MNRAS, 335, 44

Woudt, P.A., \& Warner, B. 2003, MNRAS, 340, 1011

Wynn, G.A., King, A.R., \& Horne, K. 1997, MNRAS, 286, 436 


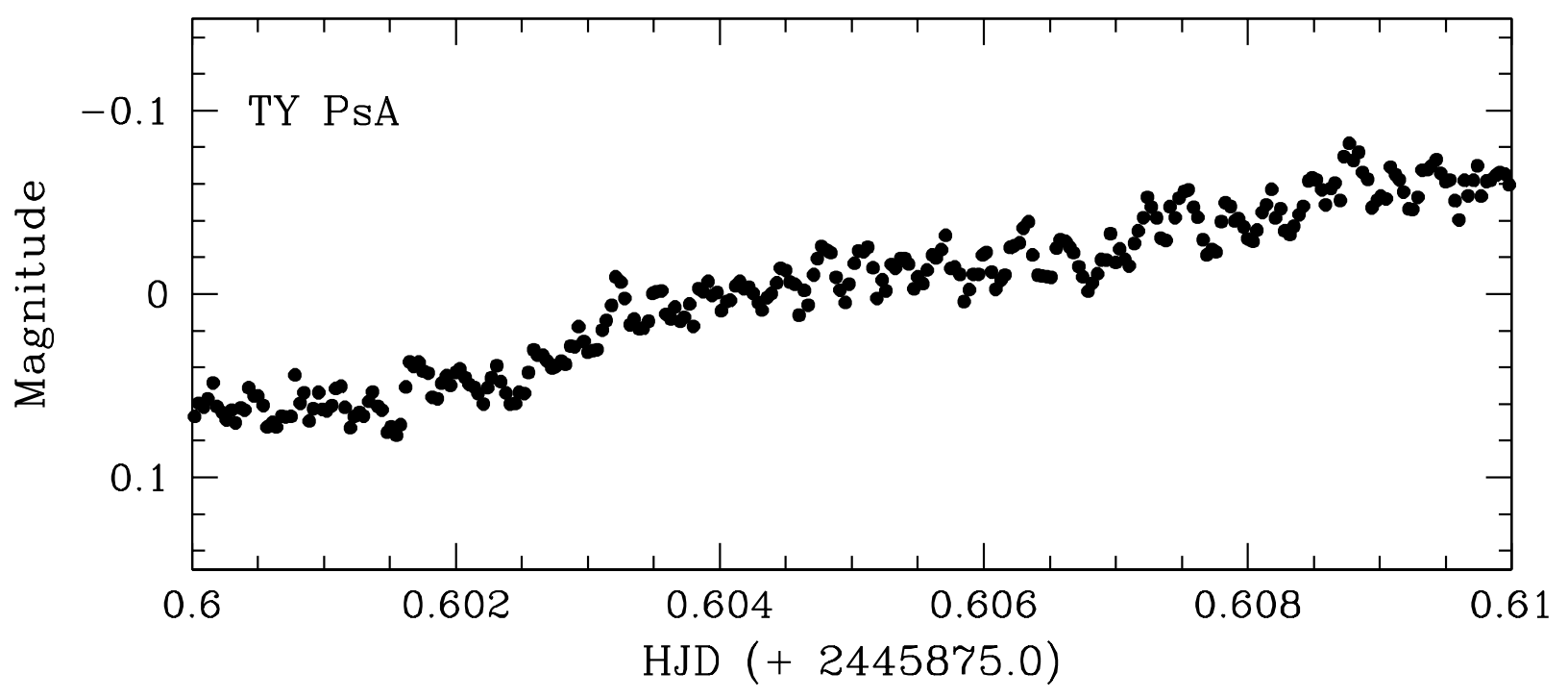

Fig. 1.- The light curve of TY PsA. The DNOs are directly visible in the light curve. Individual points are $3 \mathrm{~s}$ integrations. Adapted from Warner, O'Donoghue \& Wargau (1989). 


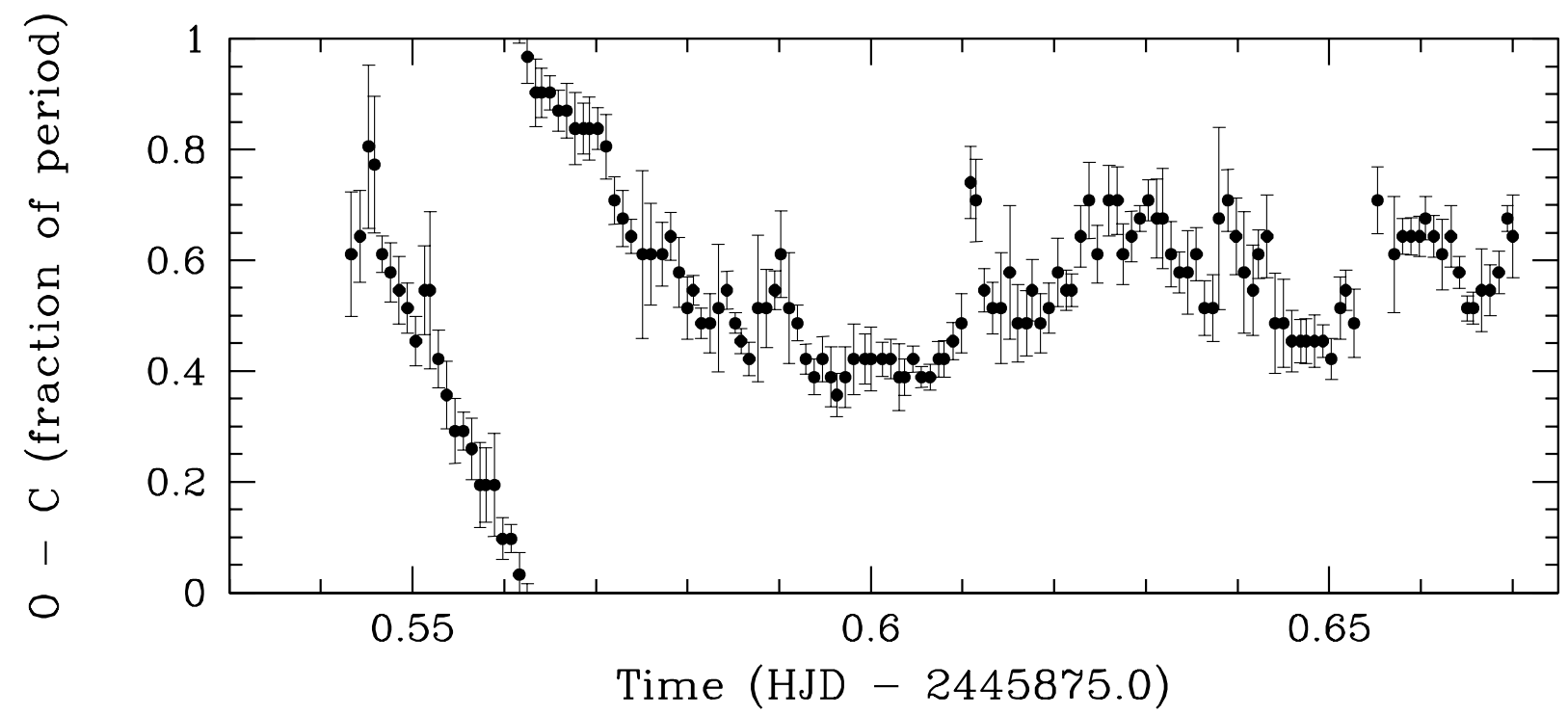

Fig. 2.- The O-C diagram of TY PsA. From observations published in Warner, O'Donoghue \& Wargau (1989). The observed oscillations are compared with a sine wave of constant period $26.64 \mathrm{~s}$. At the start of the run the period was shorter than this, giving a nearly linear change of phase $(\mathrm{O}-\mathrm{C})$, but around $0.58 \mathrm{~d}$ the period changed to average $26.64 \mathrm{~s}$, but with short and often sudden changes of phase (or, equivalent, period). The diagram "wraps round" when the phase exceeds one cycle. 


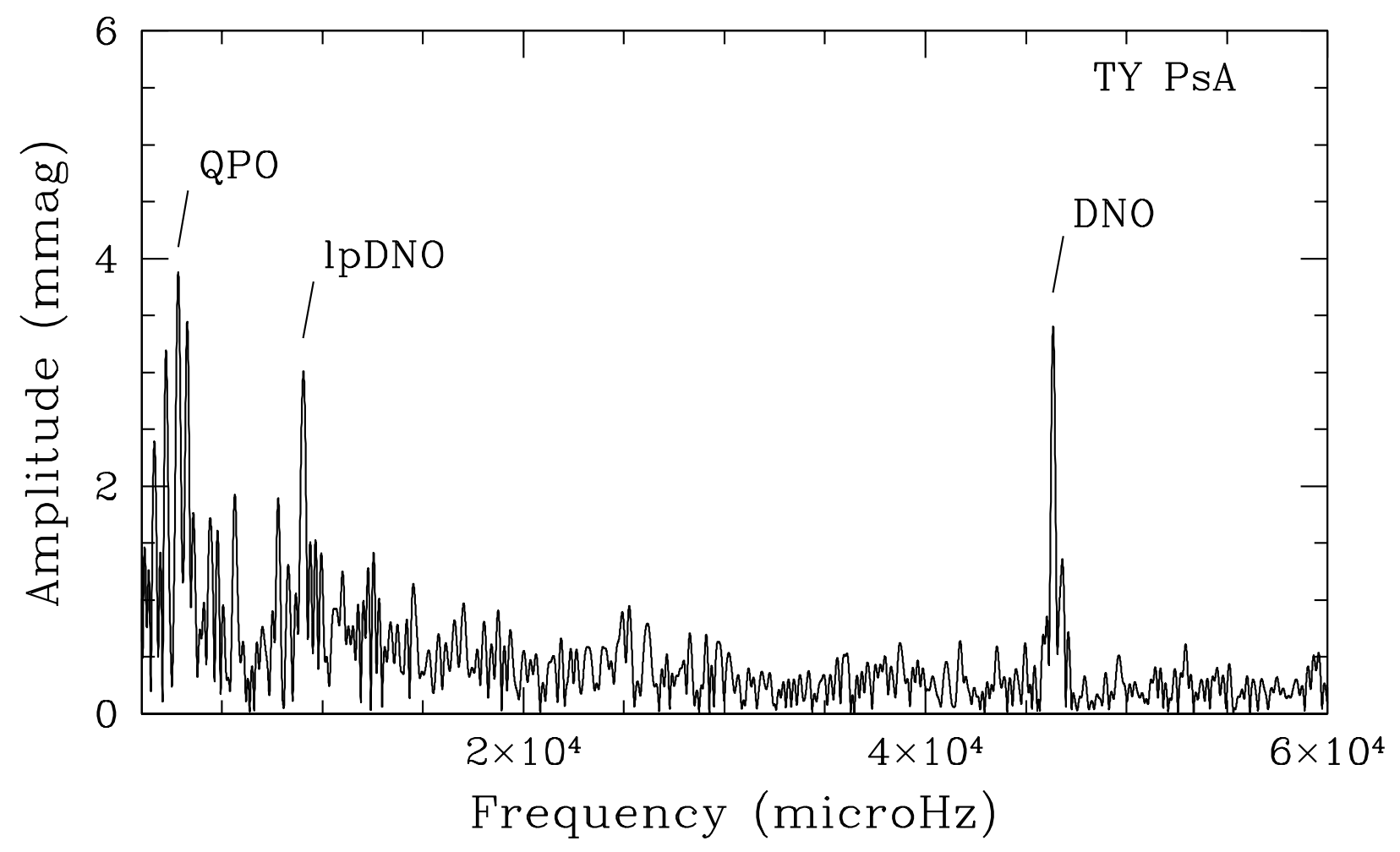

Fig. 3.- The Fourier spectrum of TY PsA. The QPO, lpDNO and DNO are all simultaneously present. From unpublished observations by M.L. Pretorius. 


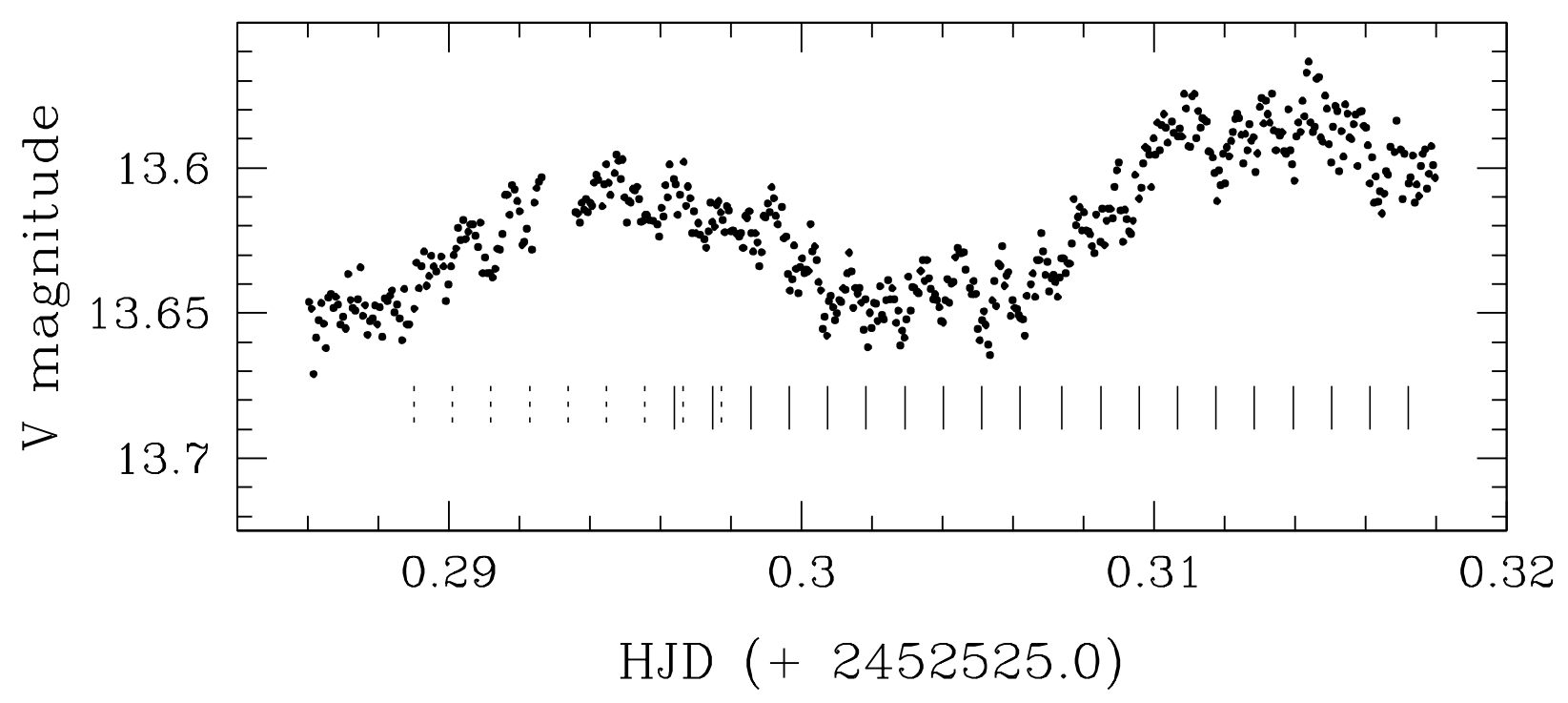

Fig. 4.- The light curve of EC 2117-54 (the first 45 minutes of run S6554). The lpDNO modulation at $94.21 \mathrm{~s}$ is clearly visible in the light curve. The lpDNO minima are marked by vertical bars. There is a phase shift around HJD 2452525.297. From Warner, Woudt \& Pretorius (2003). 


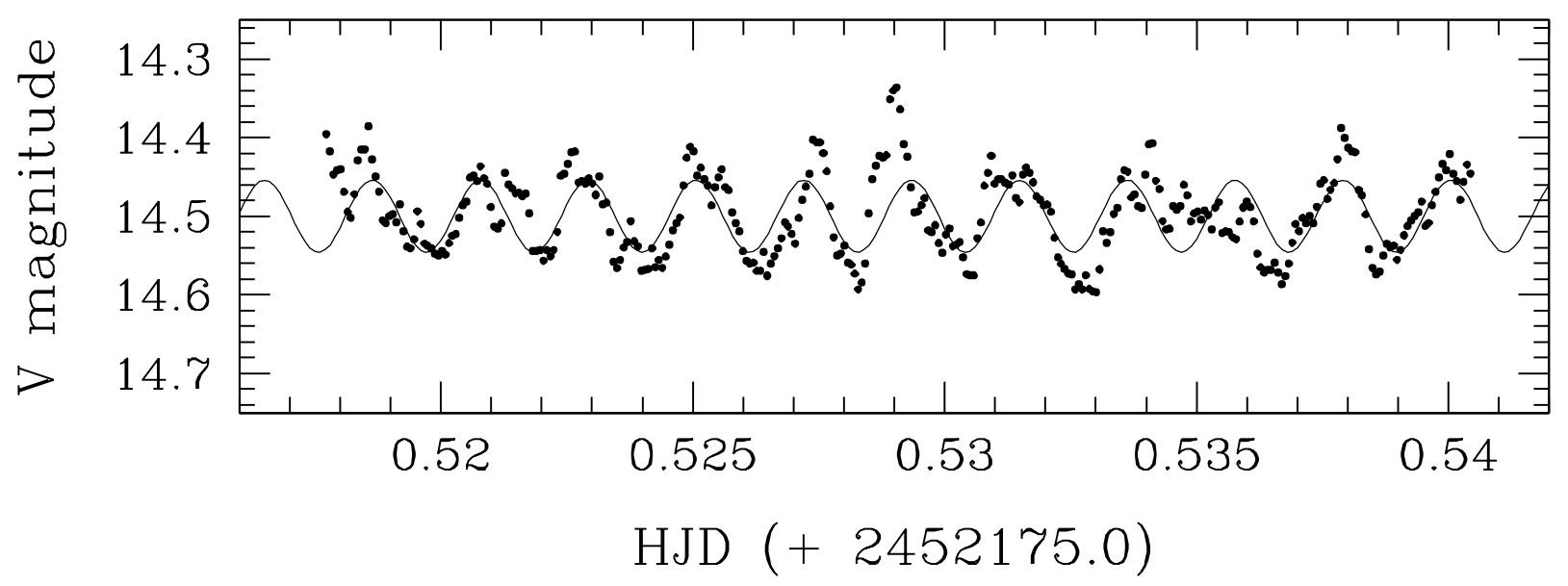

Fig. 5.- The light curve of WX Hyi, showing the 185-s QPO clearly. Superimposed is the result from the non-linear sinusoidal least-squares fit. From Warner, Woudt \& Pretorius (2003). 


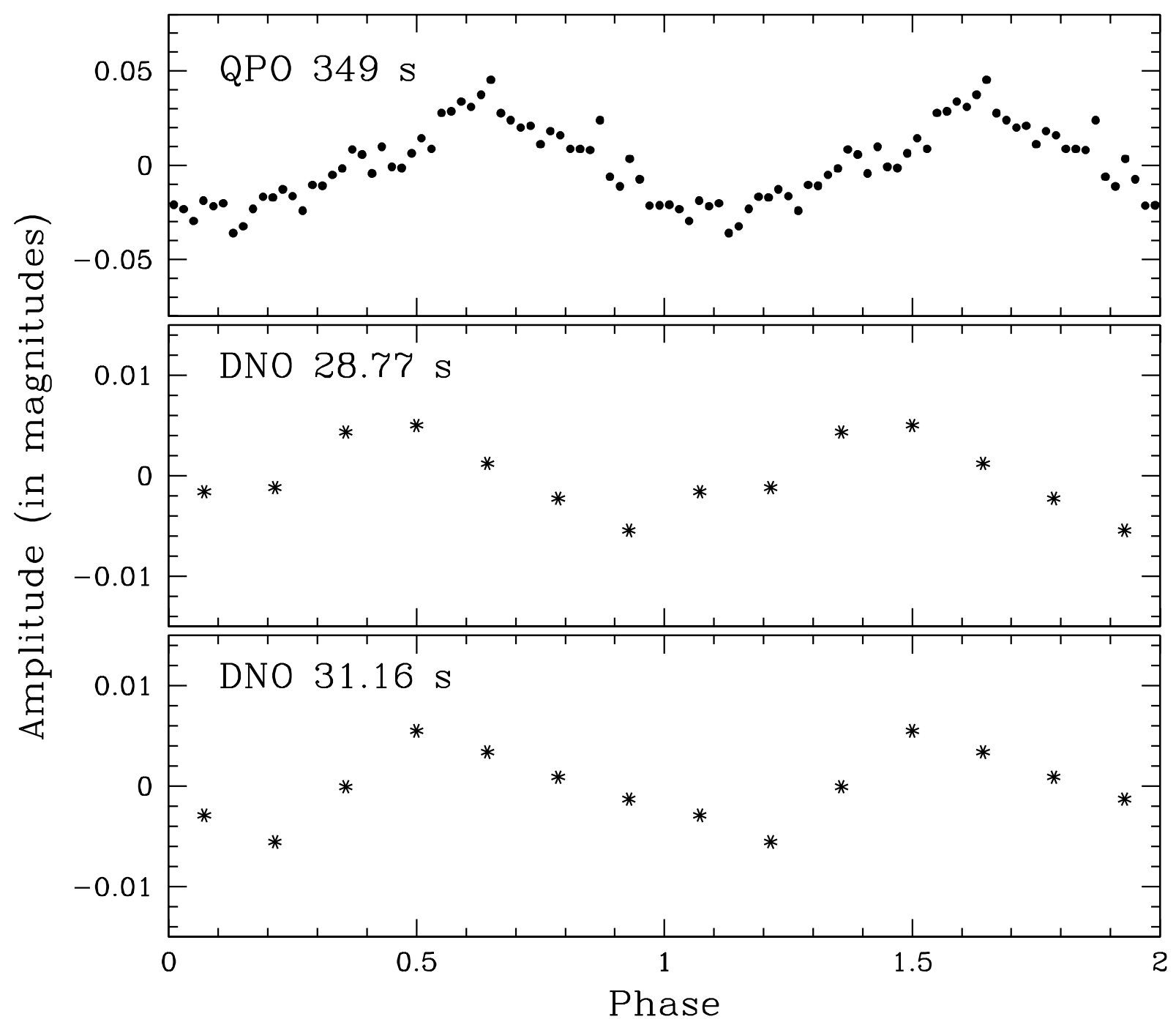

Fig. 6.- Averaged profiles of the DNOs and QPOs present simultaneously in VW Hyi (corresponding to the Fourier spectrum in Fig. 12). From Woudt \& Warner (2002a). 


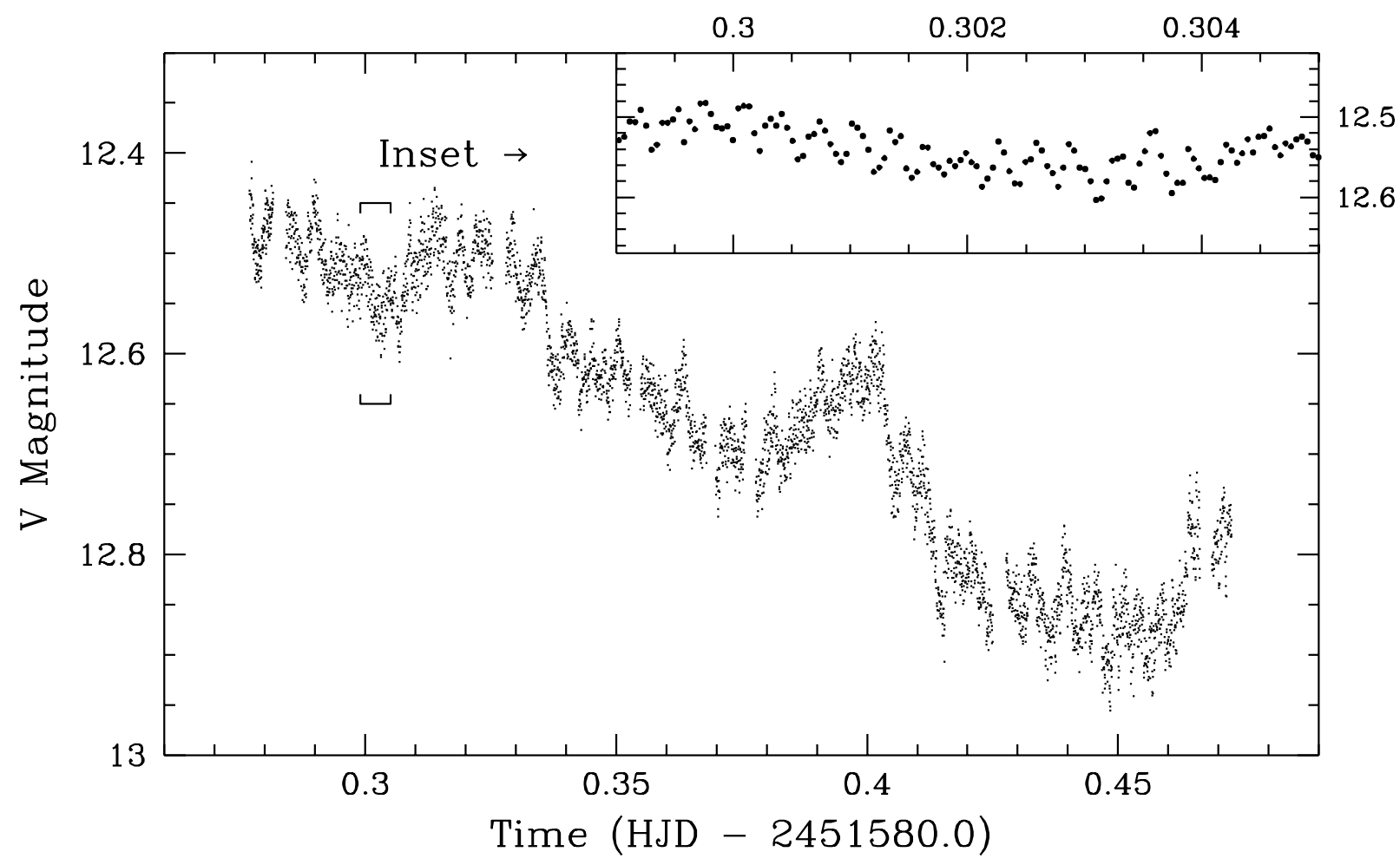

Fig. 7.- The light curve of VW Hyi on 2000 Feb 5, taken during the late decay phase of this dwarf nova outburst. The inset is an amplified view of a small part, showing the DNOs. The large humps are at the orbital period $(107 \mathrm{~min})$. QPOs are present with a range of $\sim$ $0.1 \mathrm{mag}$ and time scale $\sim 0.006 \mathrm{~d}$. From Woudt \& Warner (2002a). 


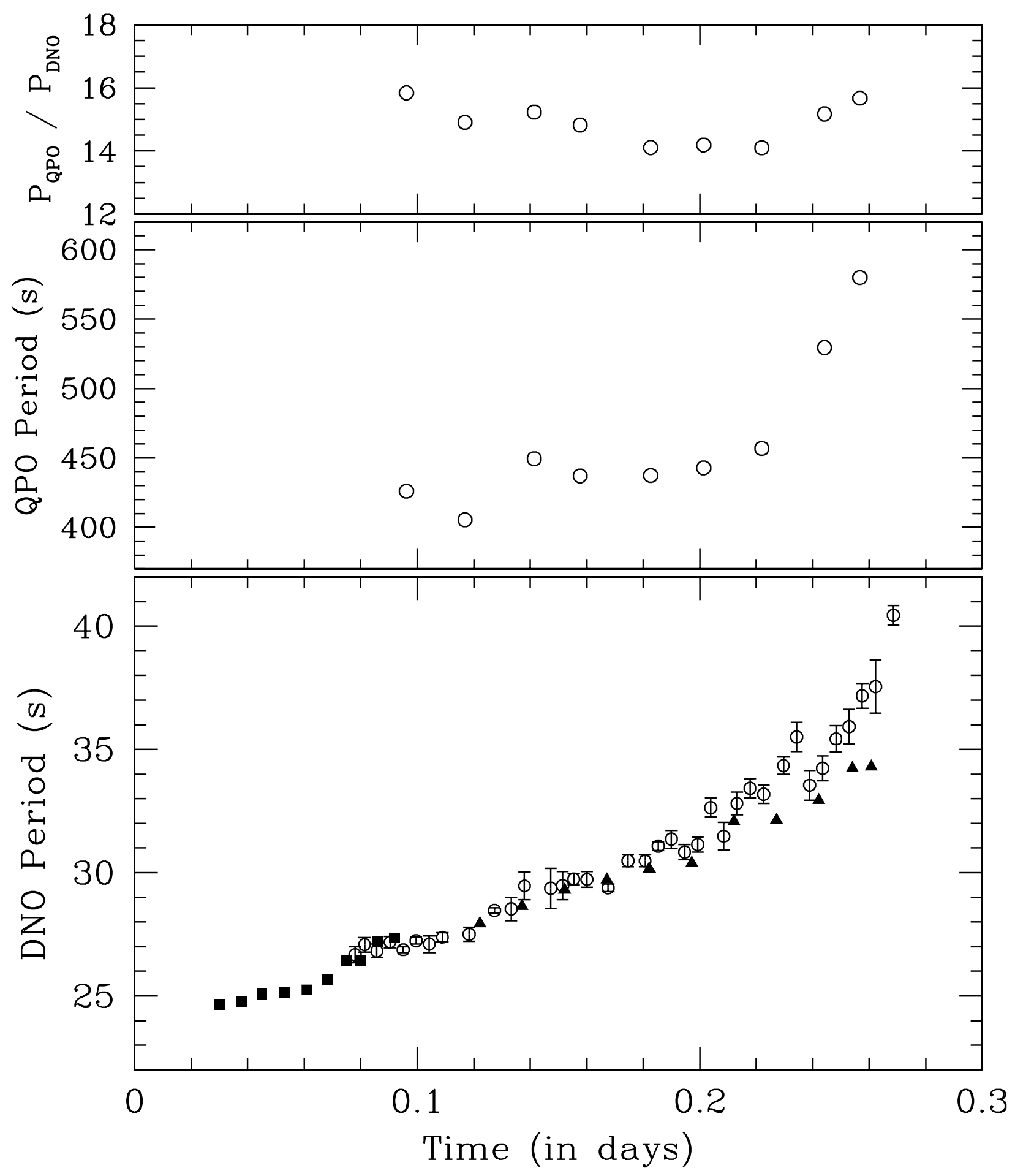

Fig. 8. - Variations with time of the DNO and QPO periods in the normal outburst of 2000 February (circles with error bars). DNOs are added for the superoutburst of 1972 December (triangles) and the normal outburst of 2001 February (squares). The topmost panel shows the ratio of the periods in the 2000 February run. From Woudt \& Warner (2002a). 


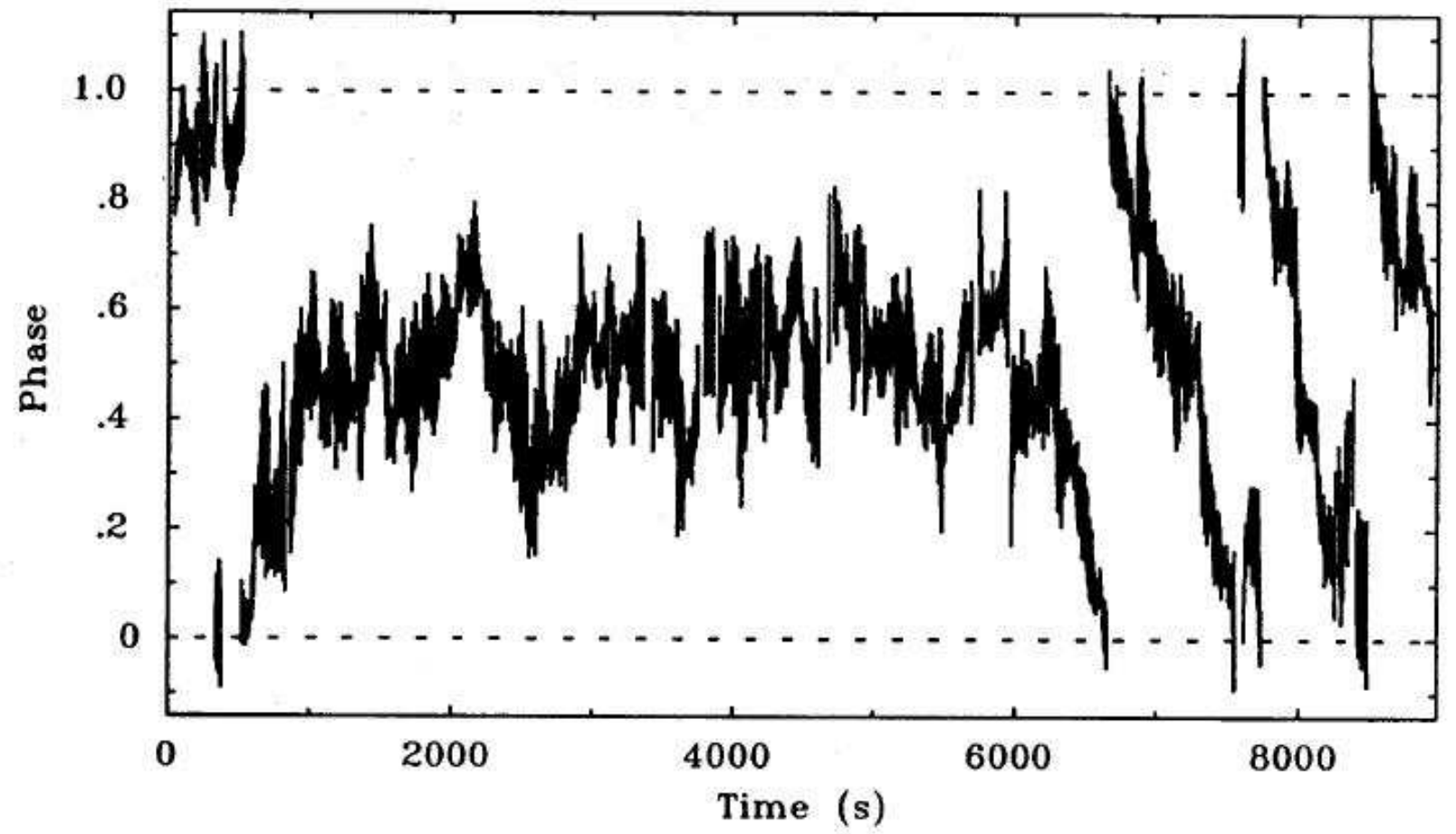

Fig. 9.- Phase behaviour of DNOs in SS Cyg in X-Rays (adapted from Jones \& Watson 1992). This is the same kind of diagram as Fig. 2, displaying $\mathrm{O}-\mathrm{C}$ phase relative to a constant period. 


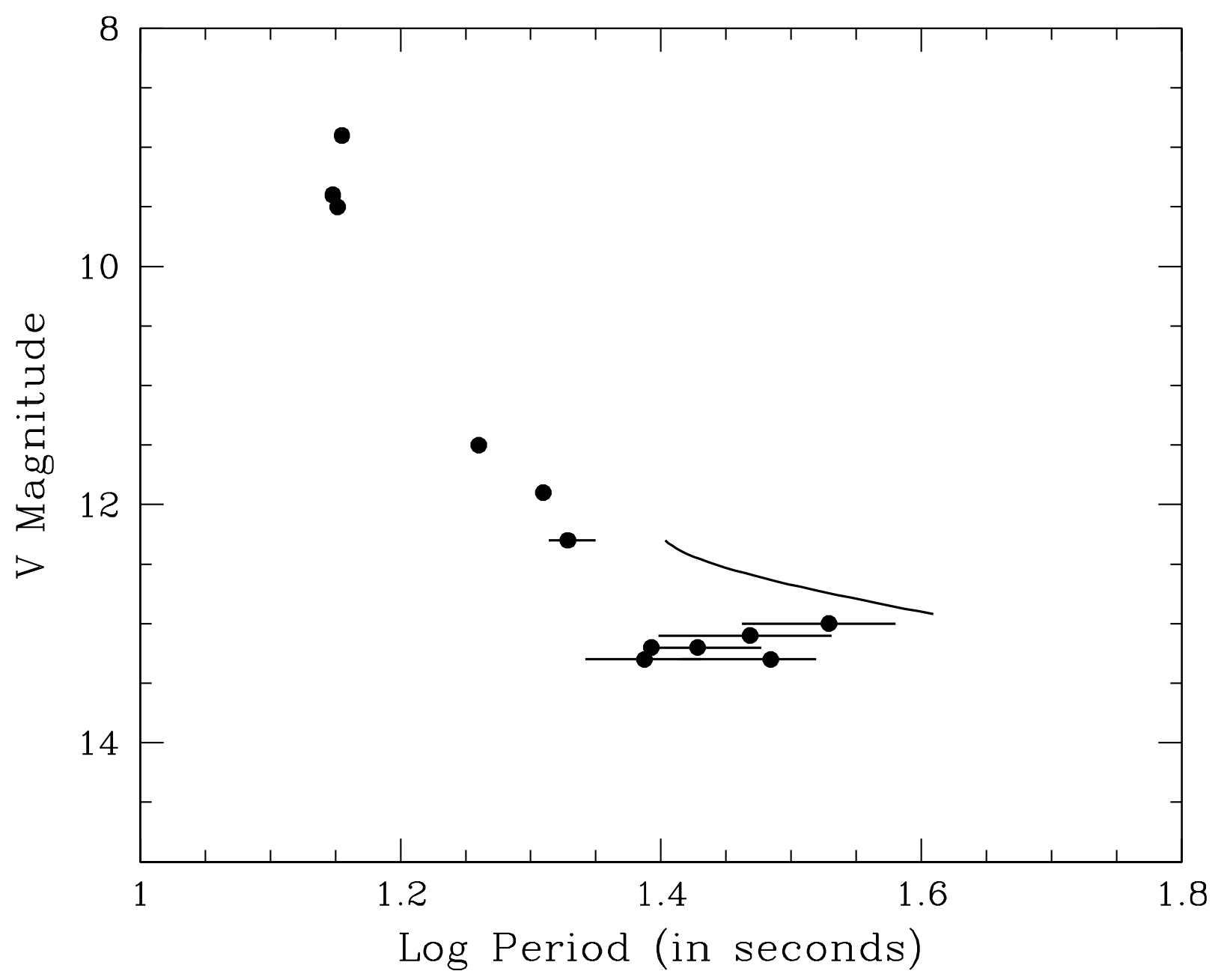

Fig. 10.- DNO periods as a function of the V magnitude of VW Hyi. The curved continuous line corresponds to the DNO evolution seen in Fig. 8. The horizontal bars show the range of DNO periods in each of the runs illustrated. From Woudt \& Warner (2002a). 


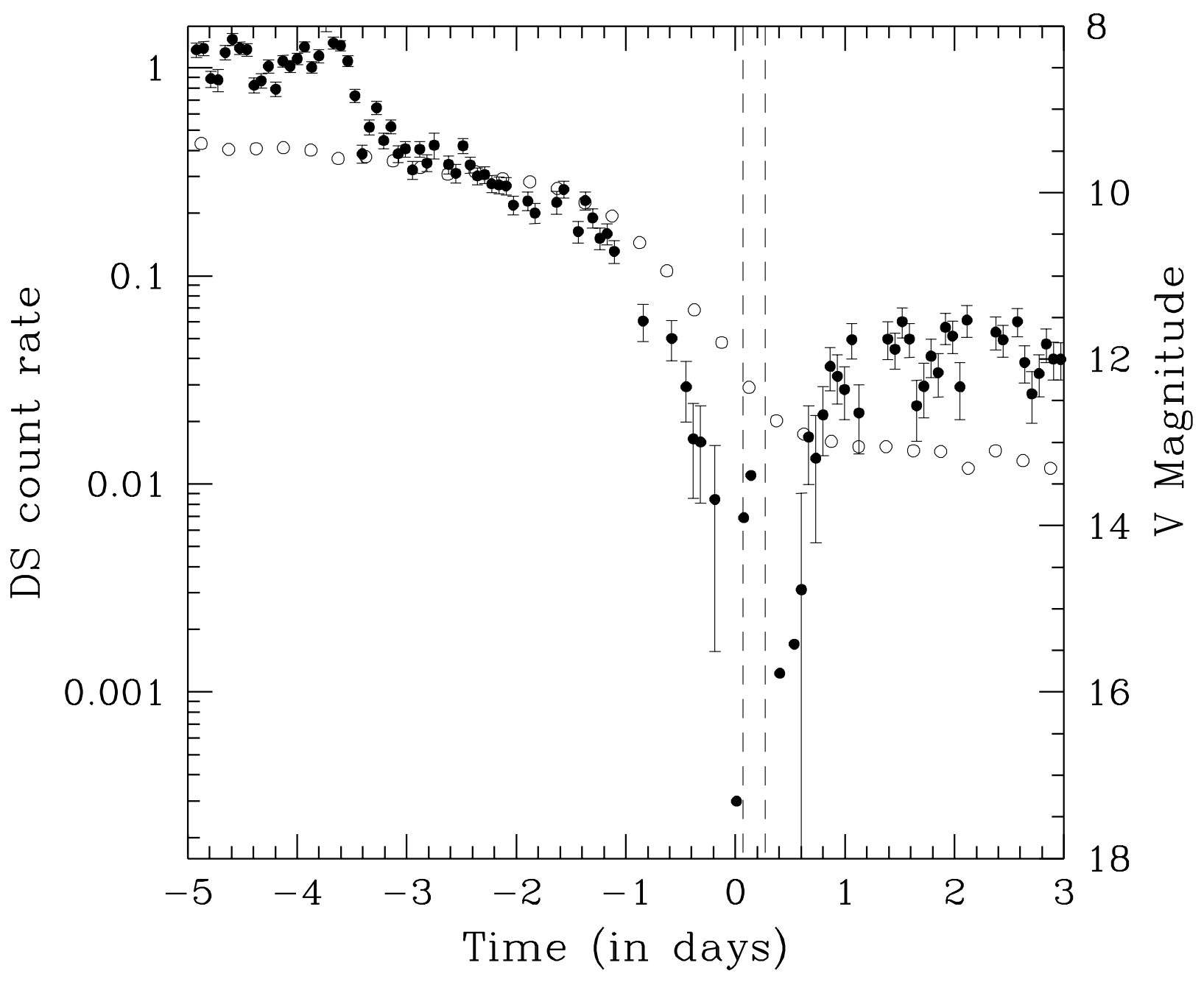

Fig. 11.- Comparison of EUV flux (DS count: filled circles) at the end of a superoutburst in VW Hyi with the average optical light curve (open circles). The vertical dashed lines show the range over which the DNOs in Fig. 8 were observed. EUV data are from C.W. Mauche (personal communication), optical data are from the Royal Astronomical Society of New Zealand (provided by F. Bateson). 


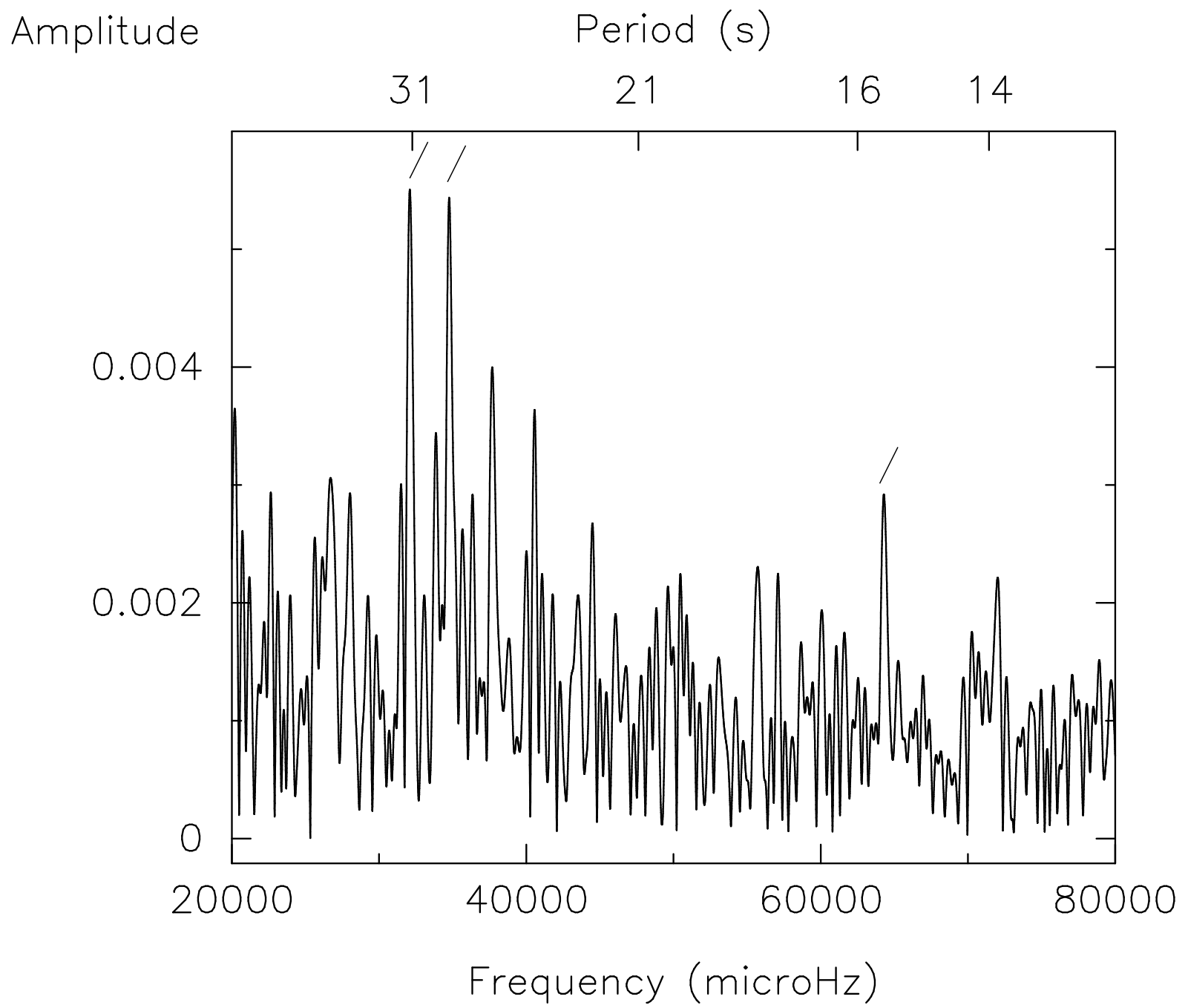

Fig. 12.- Fourier spectrum of a light curve of VW Hyi during outburst showing double DNO and harmonic to the lower frequency (indicated by bars). From Woudt \& Warner (2002a). 


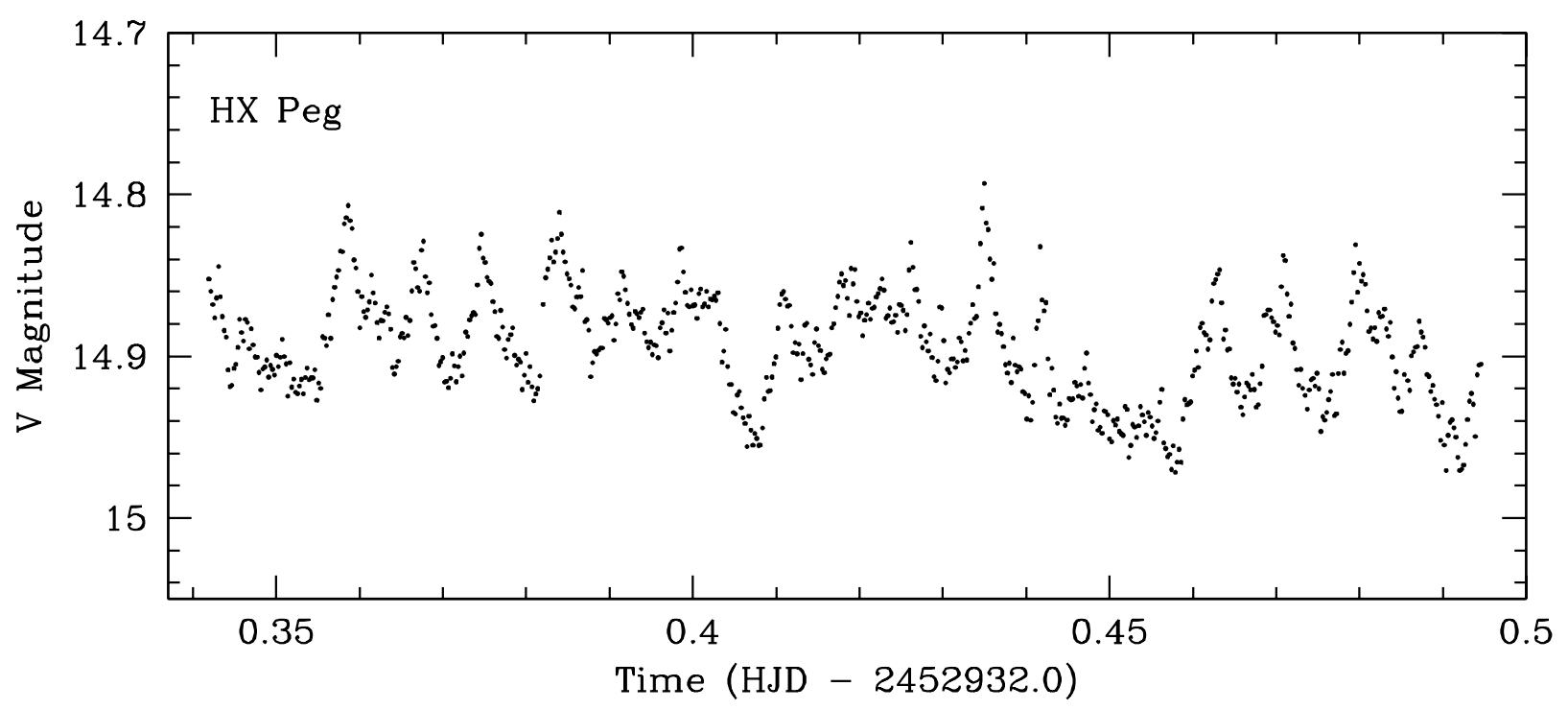

Fig. 13. - Light curve of HX Peg in outburst. The original data have been binned to $20 \mathrm{~s}$ integrations. Courtesy M.L. Pretorius. 


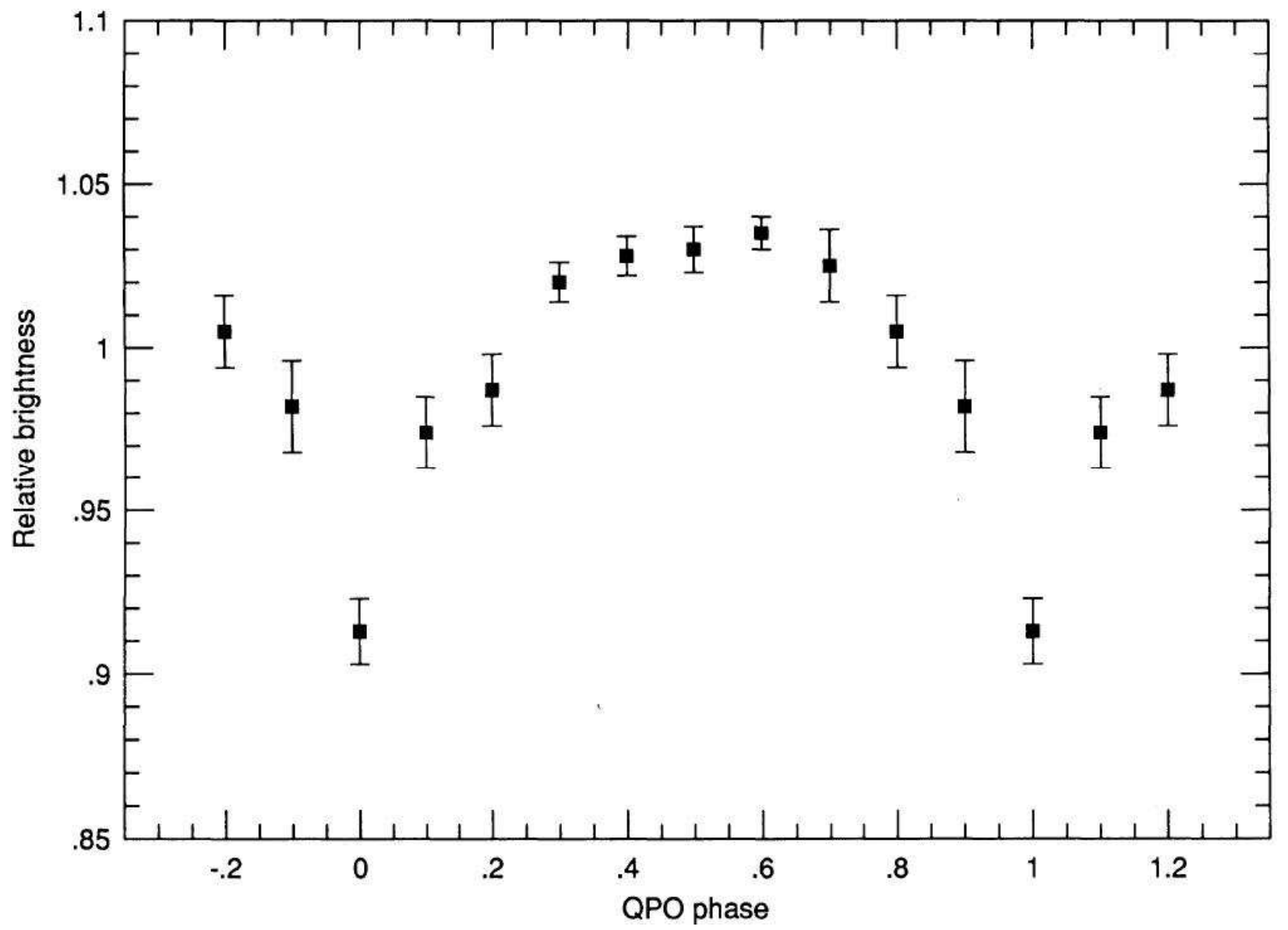

Fig. 14.- The mean light curve of the $\sim 370 \mathrm{~s}$ QPO in SW UMa (from Kato, Hirata \& Mineshige 1992). Individual cycles of the QPOs, at higher time resolution, show that the low point is caused by a shallow eclipse. 


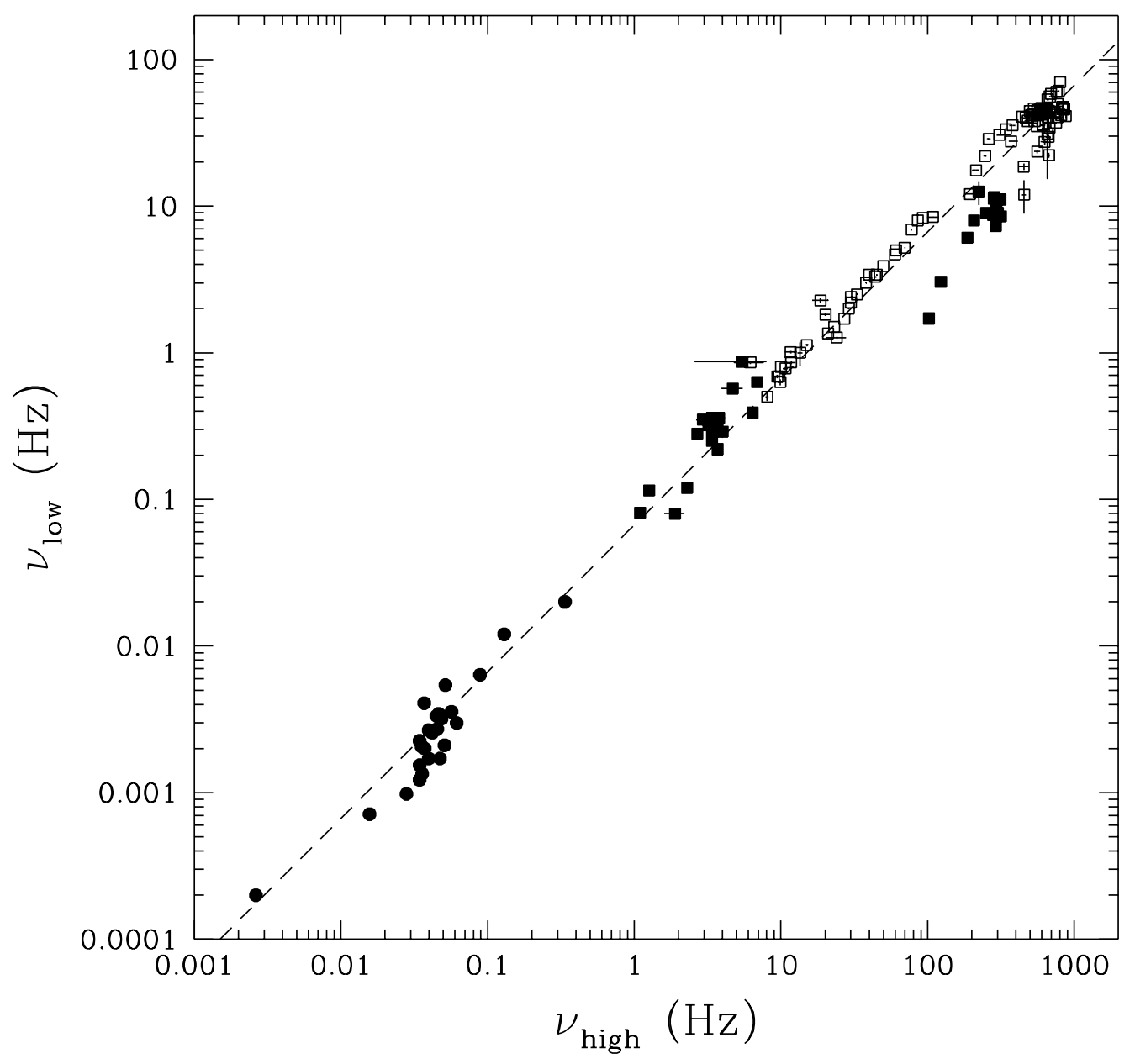

Fig. 15. - The Two-QPO diagram for X-Ray binaries (filled squares: black hole binaries, open squares: neutron star binaries) and $26 \mathrm{CVs}$ (filled circles). Each CV is only plotted once in this diagram. The X-Ray binary data are from Belloni et al. (2002) and were kindly provided by T. Belloni. The dashed line marks $P_{Q P O} / P_{D N O}=15$. From Warner, Woudt \& Pretorius (2003). 
Table 1: Rapid optical and ultraviolet modulations in cataclysmic variables.

\begin{tabular}{|c|c|c|c|c|c|c|}
\hline Star & Type & $\begin{array}{l}P_{o r b} \\
(\mathrm{~h})\end{array}$ & $\begin{array}{l}\text { DNO } \\
(\mathrm{s})\end{array}$ & $\begin{array}{c}\text { lpDNO } \\
(\mathrm{s})\end{array}$ & $\begin{array}{l}\text { QPO } \\
(\mathrm{s})\end{array}$ & References \\
\hline SS Cyg & $\mathrm{DN}$ & 6.60 & $6.58^{*}-10.9$ & $32-36$ & $83-111,730$ & $1,2,15,16,53$ \\
\hline RU Peg & $\mathrm{DN}$ & 8.99 & $11.6-11.8$ & $\rightarrow ?$ & $\sim 51$ & \\
\hline VW Hyi & NL & 1.78 & $14.03-40$ & $\sim 90$ & $400-600$ & $3,4,5$ \\
\hline EM Cyg & $\mathrm{DN}$ & 6.98 & $14.6-21.2$ & & & \\
\hline Z Cam & $\mathrm{DN}$ & 6.96 & $16.0-18.8$ & & & \\
\hline HX Peg & $\mathrm{DN}$ & 4.82 & $16.2-16.4$ & $\sim 83$ & $\sim 340,800-1900$ & 5,7 \\
\hline WX Cet & $\mathrm{DN}$ & 1.40 & 17.4 & & & 61 \\
\hline OY Car & $\mathrm{DN}$ & 1.50 & $17.6-28.0$ & $47.9,116$ & $\sim 320,1500$ & 5,6 \\
\hline WX Hyi & $\mathrm{DN}$ & 1.80 & 19.4 & & $\sim 190,1140,1560$ & 5 \\
\hline V436 Cen & $\mathrm{DN}$ & 1.50 & $19.5-20.1$ & & 475 & 4,5 \\
\hline HL Aqr & NL & 3.25 & 19.6 & & & \\
\hline RR Pic & $\mathrm{N}$ & 3.48 & $20-40$ & & & \\
\hline HT Cas & $\mathrm{DN}$ & 1.77 & $20.2-20.4$ & $\sim 100$ & & 1 \\
\hline TU Men & $\mathrm{DN}$ & 2.82 & 20.6 & & 313 & 7 \\
\hline HP Lib & $\mathrm{AMC}$ & 0.31 & & & $\sim 290$ & 59 \\
\hline CR Boo & $\mathrm{AMC}$ & 0.40 & $21-23$ & 62 & $\sim 300$ & 5 \\
\hline AQ Eri & $\mathrm{DN}$ & 1.46 & $21.0-23.5$ & $\sim 90$ & $\sim 280$ & 5 \\
\hline AH Hya & $\mathrm{DN}$ & - & 21.55 & & & 7 \\
\hline TY PsA & $\mathrm{DN}$ & 2.02 & $21.6,25.5-30$ & 110 & 355 & 7 \\
\hline BR Lup & $\mathrm{DN}$ & 1.91 & 21.65 & & & 7 \\
\hline SW UMa & $\mathrm{DN}$ & 1.36 & 22.3 & & $280-370$ & 41 \\
\hline KT Per & $\mathrm{DN}$ & 3.92 & $22.4-29.3$ & $\sim 86,147$ & & \\
\hline EC2117 & NL & 3.71 & $22.5-25.5$ & $\sim 95$ & $\sim 500$ & 5 \\
\hline WW Cet & $\mathrm{DN}$ & 4.22 & 23.1 & 103 & 263 & 7 \\
\hline SY Cnc & $\mathrm{DN}$ & 9.12 & $23.3-33.0$ & & & \\
\hline VZ Pyx & $\mathrm{DN}$ & 1.78 & 23.9 & 112 & $390, \sim 3000$ & 5,29 \\
\hline
\end{tabular}


Table 1: Continued: Rapid optical and ultraviolet modulations in cataclysmic variables.

\begin{tabular}{|c|c|c|c|c|c|c|}
\hline Star & Type & $\begin{array}{l}P_{o r b} \\
(\mathrm{~h})\end{array}$ & $\begin{array}{c}\text { DNO } \\
(\mathrm{s})\end{array}$ & $\begin{array}{c}\mathrm{lpDNO} \\
(\mathrm{s})\end{array}$ & $\begin{array}{c}\mathrm{QPO} \\
(\mathrm{s})\end{array}$ & References \\
\hline V803 Cen & $\mathrm{AMC}$ & 0.28 & & 176 & & 5 \\
\hline V1159 Ori & DN & 1.50 & $24-34$ & 177 & $\rightarrow ?$ & 7,42 \\
\hline AH Her & $\mathrm{DN}$ & 5.93 & $24.0-38.8$ & $\sim 100$ & & 1 \\
\hline CN Ori & DN & 3.91 & $24.3-32.6$ & & & 5 \\
\hline IX Vel & $\mathrm{N}$ & 4.65 & $24.6-29.1$ & & $\sim 500$ & 25 \\
\hline U Gem & DN & 4.25 & $\sim 25$ & $\sim 146$ & & 26 \\
\hline Z Cha & DN & 1.79 & $25.1-27.7$ & & 585 & 5 \\
\hline V893 Sco & DN & 1.82 & 25.2 & & $\sim 350$ & 5,30 \\
\hline BP Lyn & NL & 3.67 & 25.5 & & & \\
\hline AM CVn & $\mathrm{AMC}$ & 0.28 & 26.3 & & 290,820 & $55,56,57,58$ \\
\hline WZ Sge & DN & 1.36 & $27.87,28.95$ & & 742 & $4,8,9,10,11,12,13$ \\
\hline \multirow{3}{*}{ V2051 Oph } & & & $14.48+$ others & & & $14,31,45,46,47$ \\
\hline & DN & 1.50 & $28.06,29.77$ & & 486,1800 & 4,18 \\
\hline & & & 42.2 & & & \\
\hline UX UMa & NL & 4.72 & $28.5-30.0$ & & $\sim 650$ & 3,17 \\
\hline V3885 Sgr & NL & 4.94 & $29-32$ & & & \\
\hline HP Nor & DN & - & 35.2 & & & 7 \\
\hline RX And & DN & 5.08 & 36 & & $\sim 1000$ & 5 \\
\hline BP Cra & DN & - & 38.6 & & & 7 \\
\hline V436 Car & NL & 4.21 & $\sim 40$ & 123 & & 38 \\
\hline V533 Her & $\mathrm{N}$ & 3.52 & 63.63 & & 1400 & 5,28 \\
\hline $\mathrm{YZ} \mathrm{Cnc}$ & DN & 2.08 & & $\sim 90$ & & \\
\hline LX Ser & NL & 3.80 & $\sim 140$ & $\rightarrow ?$ & & \\
\hline X Leo & DN & 3.95 & $89-160$ & & & 7 \\
\hline TW Vir & DN & 4.38 & $112-121$ & & 1000 & 7 \\
\hline V373 Sct & $\mathrm{N}$ & - & 258.3 & $\rightarrow ?$ & & 37 \\
\hline
\end{tabular}


Table 1: Continued: Rapid optical and ultraviolet modulations in cataclysmic variables.

\begin{tabular}{|c|c|c|c|c|c|c|}
\hline Star & Type & $\begin{array}{l}P_{o r b} \\
(\mathrm{~h})\end{array}$ & $\begin{array}{c}\text { DNO } \\
(\mathrm{s})\end{array}$ & $\begin{array}{c}\text { lpDNO } \\
(\mathrm{s})\end{array}$ & $\begin{array}{c}\mathrm{QPO} \\
(\mathrm{s})\end{array}$ & References \\
\hline BT Mon & $\mathrm{N}$ & 8.01 & & & $\sim 1800$ & 5,27 \\
\hline GK Per & $\mathrm{N}$ & $1.99 \mathrm{~d}$ & $360-380$ & & 5000 & $19,20,21$ \\
\hline KR Aur & $\mathrm{DN}$ & 3.91 & & & $400-900$ & 22 \\
\hline EF Peg & NL & 1.92 & & & 400,1080 & 39 \\
\hline TV Crv & $\mathrm{DN}$ & 1.50 & & & 600 & 49 \\
\hline RW Sex & NL & 5.93 & & & 620,1280 & \\
\hline V842 Cen & $\mathrm{N}$ & - & & & $750-1300$ & 37 \\
\hline EC0528-58 & NL & - & & & $900-1560$ & 48 \\
\hline TT Ari & NL & 3.30 & & & $900-1600$ & $33,50,51$ \\
\hline V442 Cen & DN & - & & & 925 & \\
\hline V442 Oph & NL & 2.98 & & & 1000 & 50 \\
\hline RXJ 1643 & NL & 2.89 & & & 1000 & 50 \\
\hline BH Lyn & NL & 3.74 & & & 1030 & 50 \\
\hline AH Men & NL & 3.01 & & & 1100 & 50 \\
\hline V795 Her & NL & 2.60 & & & 1150 & 32,50 \\
\hline WX Ari & NL & 3.34 & & & 1180 & 50 \\
\hline SS Aur & DN & 4.39 & & & $1200-1800$ & 52 \\
\hline V751 Cyg & NL & 3.47 & & & 1230 & 34 \\
\hline LS Peg & NL & 4.20 & & & 1240 & $35,36,40$ \\
\hline NSV 10934 & $\mathrm{DN}$ & 1.7 & & & 1300 & 60 \\
\hline V426 Oph & $\mathrm{DN}$ & 6.85 & & & 1680 & \\
\hline GO Com & DN & 1.58 & & & 1980 & \\
\hline V592 Cas & $\mathrm{DN}$ & 2.76 & & & 2160 & 44 \\
\hline CW Mon & $\mathrm{DN}$ & 4.23 & & & 2200 & 54 \\
\hline SU UMa & $\mathrm{DN}$ & 1.83 & & & 2280 & \\
\hline MV Lyr & NL & 3.20 & & & $\sim 3000$ & 23,24 \\
\hline
\end{tabular}


Notes Table 1: $\mathrm{DN}=$ dwarf nova, $\mathrm{N}=$ nova, $\mathrm{NL}=$ nova-like, AMC $=\mathrm{AM} \mathrm{CVn}$ star,${ }^{*} \mathrm{~A}$ period approximately half this was also observed.

The References are additional to those given in Table 8.2 of Warner (1995).

1 Patterson 1981; 2 Mauche \& Robinson 2001; 3 Woudt \& Warner 2002a; 4 Warner \& Woudt 2002; 5 Warner, Woudt \& Pretorius 2003; 6 Marsh \& Horne 1998; 7 M. L. Pretorius (unpublished); 8 Knigge et al. 2002; 9 Skidmore et al. 2002; 10 Skidmore et al. 1999; 11 Patterson et al. 1998; 12 Welsh et al. 1997; 13 Skidmore et al. 1997; 14 Provencal \& Nather 1997; 15 Mauche 1996a; 16 Mauche 1997a; 17 Knigge et al. 1998; 18 Steeghs et al. 2001; 19 Nogami, Kato \& Baba 2002; 20 Morales-Rueda, Still \& Roche 1996; 21 Morales-Rueda, Still \& Roche 1999; 22 Kato et al. 2002; 23 Pavlenko \& Shugarov 1999; 24 Kraicheva et al. 1999a; 25 Williams \& Hiltner 1984; 26 Long et al. 1996; 27 Smith, Dhillon \& Marsh 1998; 28 Rodriguez-Gil \& Martinez-Pais 2002; 29 Remillard et al. 1994; 30 Bruch, Steiner \& Gneiding 2000; 31 Patterson et al. 2002a; 32 Rodriguez-Gil et al. 2002; 33 Kraicheva et al. 1999b; 34 Patterson et al. 2001; 35 Taylor et al. 1999; 36 Rodriguez-Gil et al. 2001; 37 Woudt \& Warner 2003; 38 Woudt \& Warner 2002b; 39 Kato 2002; 40 Szkody et al. 2001; 41 Nogami et al. 1998; 42 Patterson et al. 1995. 43 Skillman, Patterson \& Thorstensen 1995; 44 Kato \& Starkey 2002; 45 Provencal \& Nather 1997; 46 Araujo-Betancor 2003; 47 Welsh et al. 2003; 48 Chen et al. 2001; 49 Uemura et al. 2001; 50 Patterson et al. 2002b; 51 Tremko et al. 1996; 52 Tovmassian 1988; 53 Mauche 2002; 54 Kato et al. 2003; 55 Patterson et al. 1979; 56 Skillman et al. 1999; 57 Patterson et al. 1992; 58 Provencal et al. 1995; 59 Patterson et al. 2002c; 60 Kato et al. 2004; 61 J. Patterson (private communication). 
Table 2: Rapid oscillations in X-Rays.

\begin{tabular}{|c|c|c|c|c|c|c|}
\hline Star & Type & $\begin{array}{l}P_{\text {orb }} \\
(\mathrm{h})\end{array}$ & $\begin{array}{l}\text { Periods } \\
\text { (s) }\end{array}$ & Energy & State & References \\
\hline \multirow[t]{3}{*}{ SS Cyg } & $\mathrm{DN}$ & 6.60 & $7.4-10.7$ & Soft & $\mathrm{O}$ & $1-3,9$ \\
\hline & & & $2.8^{*}$ & Soft & $\mathrm{O}$ & 10 \\
\hline & & & $155-245$ & Hard & $\mathrm{O}$ & 17 \\
\hline \multirow[t]{4}{*}{ VW Hyi } & $\mathrm{DN}$ & 1.78 & $14.06,14.2-14.4$ & Soft & $\mathrm{O}$ & 6 \\
\hline & & & $63-68$ & Soft & $\mathrm{O}$ & 6 \\
\hline & & & $\sim 60$ & Hard & $\mathrm{Q}$ & 14 \\
\hline & & & $\sim 500$ & Hard & $\mathrm{O}$ & 12 \\
\hline HT Cas & $\mathrm{DN}$ & 1.77 & 21.85: & Hard & Q & 4,7 \\
\hline \multirow[t]{3}{*}{ U Gem } & $\mathrm{DN}$ & 4.25 & $25-29$ & Soft & $\mathrm{O}$ & 2,8 \\
\hline & & & 121,135 & Hard & Q & 7 \\
\hline & & & 585 & Hard & $\mathrm{O}$ & 7 \\
\hline WZ Sge & $\mathrm{DN}$ & 1.36 & 27.8 & Hard & Q & 13 \\
\hline SU UMa & $\mathrm{DN}$ & 1.83 & 33.93: & Hard & Q & 4 \\
\hline YZ Cnc & $\mathrm{DN}$ & 2.21 & 222 & Hard & Q & 7 \\
\hline RW Sex & $\mathrm{NL}$ & 5.93 & 254 & Hard & & 7 \\
\hline AB Dra & $\mathrm{DN}$ & 3.65 & 290 & Hard & $\mathrm{O}$ & 7 \\
\hline OY Car & $\mathrm{DN}$ & 1.50 & 2240 & Soft & Q & 11 \\
\hline GK Per & $\mathrm{N}, \mathrm{DN}$ & $1.99 \mathrm{~d}$ & $3000-5000$ & Hard & $\mathrm{O}$ & 15,16 \\
\hline
\end{tabular}

Notes Table 2: $\mathrm{O}=$ outburst, $\mathrm{Q}=$ quiescence. A colon (:) denotes a less certain observation. ${ }^{*}$ Frequency doubling had occurred.

1 Cordova et al. 1980; 2 Cordova et al. 1984; 3 Watson, King \& Heise 1985; 4 Eracleous, Patterson \& Halpern 1991; 5 Jensen et al. 1983; 6 van der Woerd, Heise \& Bateson 1986; 7 Cordova \& Mason 1984; 8 Mason et al. 1988; 9 Jones \& Watson 1992; 10 van Teeseling 1997; 11 Ramsay et al. 2001; 12 Wheatley et al. 1996; 12 Wheatley et al. 1996; 13 Patterson et al. 1998; 14 Pandel, Cordova \& Howell 2003; 15 Watson, King \& Osborne 1985; 16 Ishida et al. 1996; 17 Wheatley, Mauche \& Mattei 2003. 